SANDIA REPORT

SAND90-2318 - UC-920

Unlimited Release

Printed May 1995
RECEIVED

JUN 191995

OSTI

\title{
Deformation Study of Separator Pellets for Thermal Batteries
}

Ronald A. Guidotti, Frederick W. Reinhardt, Edward V. Thomas

\author{
Prepared by \\ Sandia National Laboratories \\ Albuquerque, New Mexico 87185 and Livermore, California 94550 \\ for the United States Department of Energy \\ under Contract DE-AC04-94AL85000
}

Approved for public release; distribution is unlimited.

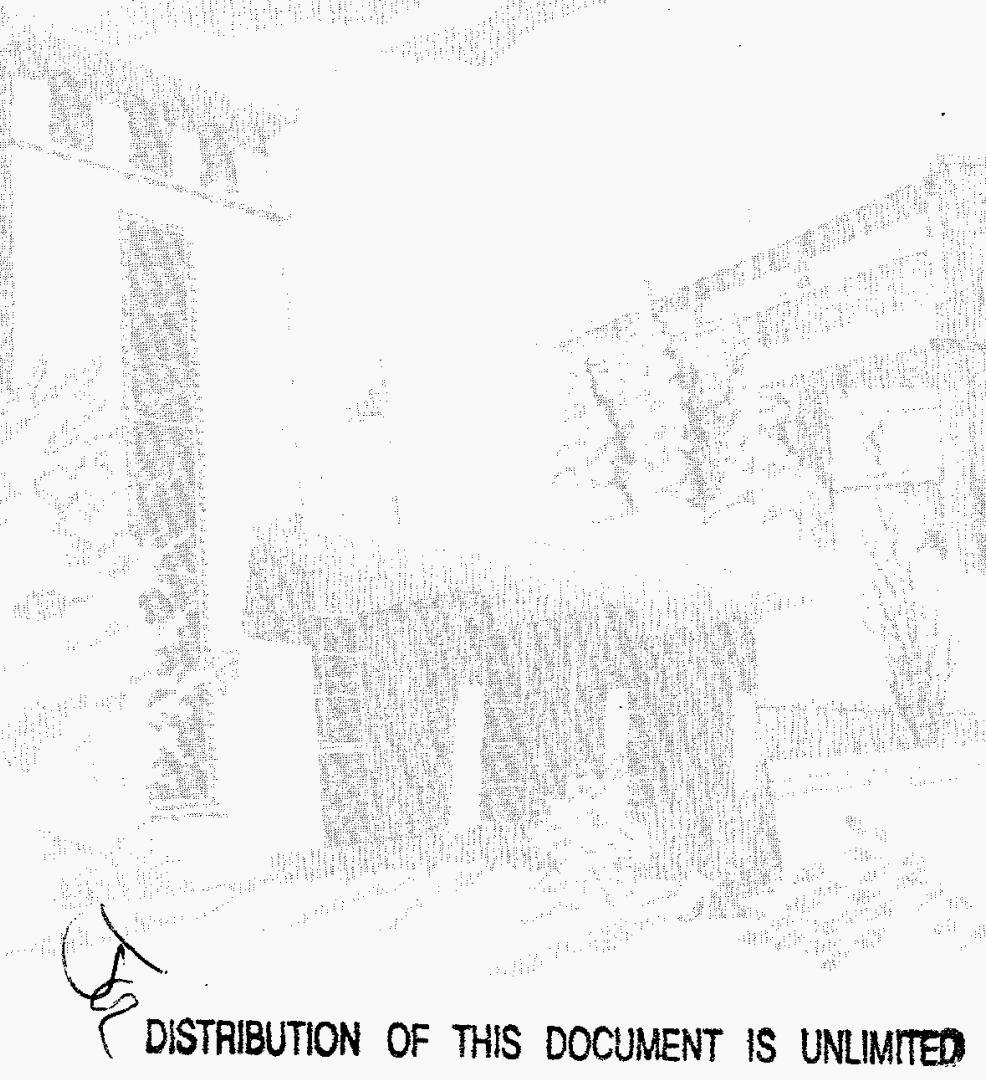


Issued by Sandia National Laboratories, operated for the United States Department of Energy by Sandia Corporation.

NOTICE: This report was prepared as an account of work sponsored by an agency of the United States Government. Neither the United States Government nor any agency thereof, nor any of their employees, nor any of their contractors, subcontractors, or their employees, makes any warranty, express or implied, or assumes any legal liability or responsibility for the accuracy, completeness, or usefulness of any information, apparatus, product, or process disclosed, or represents that its use would not infringe privately owned rights. Reference herein to any specific commercial product, process, or service by trade name, trademark, manufacturer, or otherwise, does not necessarily constitute or imply its endorsement, recommendation, or favoring by the United States Government, any agency thereof or any of their contractors or subcontractors. The views and opinions expressed herein do not necessarily state or reflect those of the United States Government, any agency thereof or any of their contractors.

Printed in the United States of America. This report has been reproduced directly from the best available copy.

Available to DOE and DOE contractors from

Office of Scientific and Technical Information

PO Box 62

Oak Ridge, TN 37831

Prices available from (615) 576-8401, FTS 626-8401

Available to the public from

National Technical Information Service

US Department of Commerce

5285 Port Royal Rd

Springfield, VA 22161

NTIS price codes

Printed copy: A04

Microfiche copy: A01 


\section{DISCLAIMER}

Portions of this document may be illegible in electronic image products. Images are produced from the best available original document. 
SAND90-2318

Distribution

Unlimited Release

Printed May 1995

Category UC-920

\title{
Deformation Study of Separator Pellets for Thermal Batteries
}

\author{
Ronald A. Guidotti and Frederick W. Reinhardt \\ Battery Research Departtment
}

Edward V. Thomas

Statistics, Computing, and Human Factors Department

Sandia National Laboratories

Albuquerque, New Mexico 87185-5800

\begin{abstract}
The deformation characteristics of pellets of electrolyte-binder (EB) mixes based on $\mathrm{MgO}$ were measured under simulated, thermal-battery conditions. Measurements (using a statistically designed experimental strategy) were made as a function of applied pressure, temperature, and percentage of theoretical density for four molten-salt electrolytes at two levels of MgO. The EB mixes are used as separators in Li-alloy thermal batteries. The electrolytes included LiCl-KCl eutectic, $\mathrm{LiCl}-\mathrm{LiBr}-\mathrm{KBr}$ eutectic, $\mathrm{LiBr}-\mathrm{KBr}-\mathrm{LiF}$ eutectic, and a $\mathrm{LiCl}-\mathrm{LiBr}-\mathrm{LiF}$ electrolyte with a minimum-melting composition. The melting points ranged from $313^{\circ} \mathrm{C}$ to $436^{\circ} \mathrm{C}$. The experimental data were used to develop statistical models that approximate the deformation behavior of pellets of the various EB mixes over the range of experimental conditions we examined. In this report, we discuss the importance of the deformation response surfaces to thermal-battery design.

OSTRIBUTION OF THIS DOCUMENT IS UNLIMITED
\end{abstract}




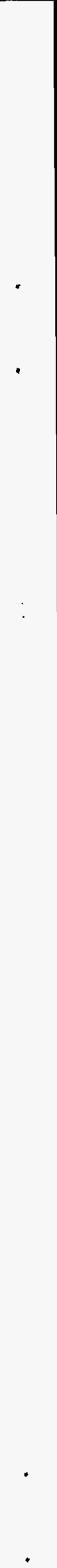




\section{Contents}

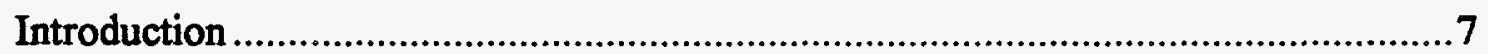

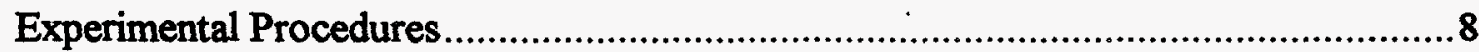

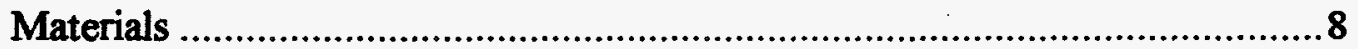

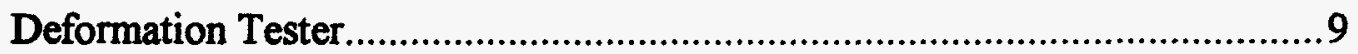

Statistical Experimental Design ..................................................................

Statistical Analysis and Modeling ..............................................................

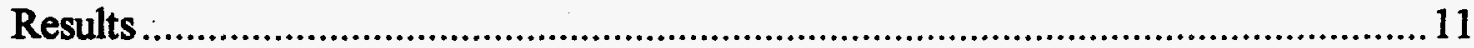

$\mathrm{LiCl}-\mathrm{KCl}$ (Standard) Electrolyte: $35 \% \mathrm{MgO}$..............................................11

LiCl-KCl (Standard) Electrolyte: $40 \%$ MgO ............................................13

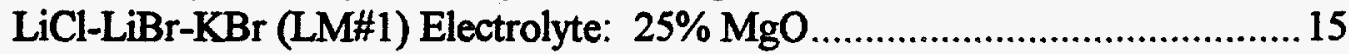

LiCl-LiBr-KBr (LM\#1) Electrolyte: $30 \%$ MgO .....................................16

LiBr-KBr-LiF (LM\#2) Electrolyte: $25 \% \mathrm{MgO}$...........................................17

Li-Br-KBr-LiF (LM\#2) Electrolyte: 30\% MgO ....................................... 19

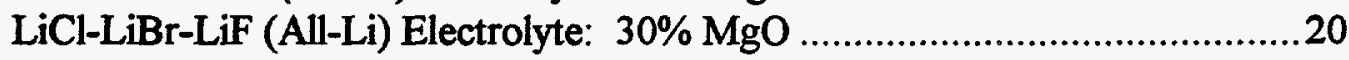

LiCl-LiBr-LiF (All-Li) Electrolyte: $35 \% \mathrm{MgO}$.........................................21

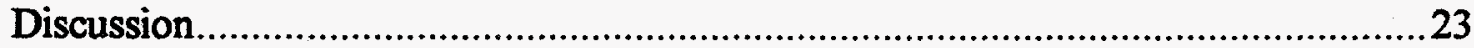

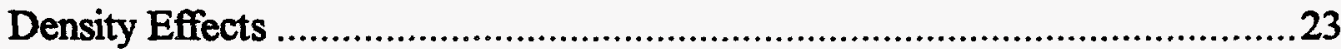

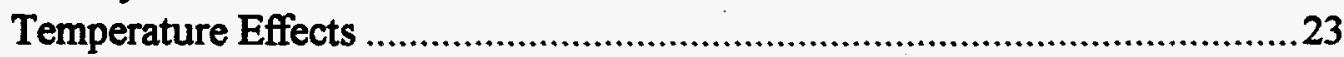

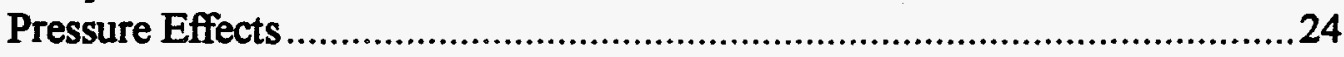

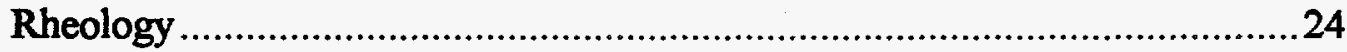

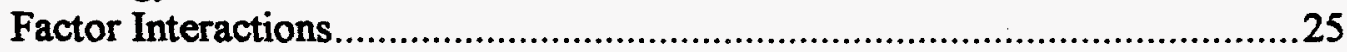

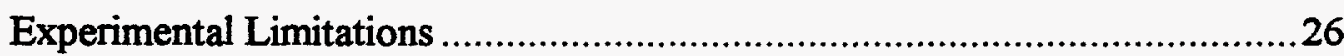

Optimum EB Densities and Battery-Design Implications ..............................26

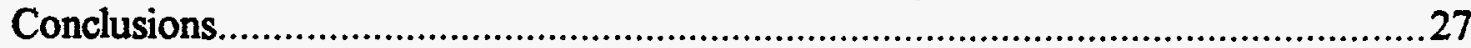

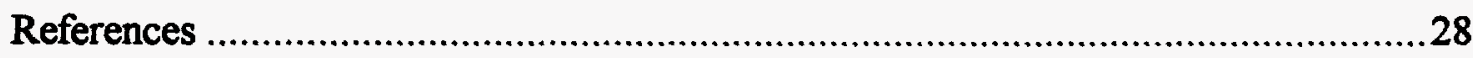

\section{Appendices}

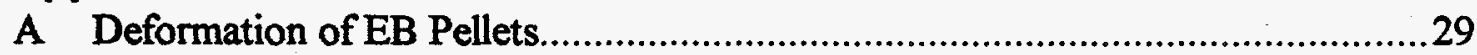

B Statistical Models for Deformation of EB Pellets.............................................35

C Comparison of Predicted and Experimental Values for the Deformation of EB Pellets

\section{Figures}

1 Cross-Sectional Representation of a Thermal Cell .......................................

2 Representative Deformation Profile at $530^{\circ} \mathrm{C}$ and 14.3 Psig Applied

Pressure of Separator Pellet Based on LiCl- $\mathrm{KCl}$ Eutectic and 35\% MgO........ 12

3 Contour Plot for Estimated Deformation of EB Pellets Based on LiCl-KCl Eutectic and $35 \% \mathrm{MgO}$ as a Function of Applied Pressure and \% Theoretical Density for Temperatures of $500^{\circ} \mathrm{C}-560^{\circ} \mathrm{C}$ 
4 Contour Plot for Estimated Deformation of EB Pellets Based on $\mathrm{LiCl}-\mathrm{KCl}$ Eutectic and $40 \% \mathrm{MgO}$ as a Function of Applied Pressure and $\%$ Theoretical Density for Temperatures of $500^{\circ} \mathrm{C}-560^{\circ} \mathrm{C}$

5 Contour Plot for Estimated Deformation of EB Pellets Based on $\mathrm{LiCl}-\mathrm{LiBr}-\mathrm{KBr}$ Eutectic and $25 \% \mathrm{MgO}$ as a Function of Applied Pressure and \% Theoretical Density for Several Temperatures
A. $500^{\circ} \mathrm{C}$.

B. $560^{\circ} \mathrm{C}$

Contour Plot for Estimated Deformation of EB Pellets Based on $\mathrm{LiCl}-\mathrm{LiBr}-\mathrm{KBr}$ Eutectic and $30 \% \mathrm{MgO}$ as a Function of Applied Pressure and \% Theoretical Density for Several Temperatures
A. $500^{\circ} \mathrm{C}$. 16

B. $560^{\circ} \mathrm{C}$

Contour Plot for Estimated Deformation of EB Pellets Based on

$\mathrm{LiBr}-\mathrm{KBr}-\mathrm{LiF}$ Eutectic and $25 \% \mathrm{MgO}$ as a Function of Applied

Pressure and \% Theoretical Density for Several Temperatures
A. $5000^{\circ} \mathrm{C}$.
B. $5600^{\circ} \mathrm{C}$

8 Contour Plot for Estimated Deformation of EB Pellets Based on

$\mathrm{LiBr}-\mathrm{KBr}-\mathrm{LiF}$ Eutectic and $30 \% \mathrm{MgO}$ as a Function of Applied

Pressure and \% Theoretical Density for Several Temperatures
A. $5000^{\circ} \mathrm{C}$
B. $560^{\circ} \mathrm{C}$

9 Contour Plot for Estimated Deformation of EB Pellets Based on

$\mathrm{LiCl}-\mathrm{LiBr}-\mathrm{LiF}$ Electrolyte and $30 \% \mathrm{MgO}$ as a Function of Applied

Pressure and \% Theoretical Density for Several Temperatures
A. $5000^{\circ} \mathrm{C}$. 20

B. $560^{\circ} \mathrm{C}$

10 Contour Plot for Estimated Deformation of EB Pellets Based on

$\mathrm{LiCl}-\mathrm{LiBr}-\mathrm{LiF}$ Electrolyte and $35 \% \mathrm{MgO}$ as a Function of Applied

Pressure and \% Theoretical Density for Several Temperatures
A. $500^{\circ} \mathrm{C}$
B. $560^{\circ} \mathrm{C}$

\section{Tables}

$1 \quad$ Electrolytes Evaluated in Separator Study .................................................9

2 Experimental Factors and Associated Levels ............................................ 10

3 Terms Used for Modeling Deformation Data ........................................... 11

4 Deformation of Separator Pellets Based on LiCl-KCl Eutectic and 35\%

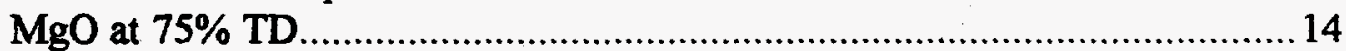

5 Deformation of Separator Pellets Based on LiCl-KCl Eutectic and $40 \%$

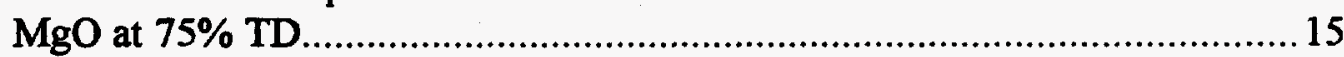


6 Deformation of Separator Pellets Based on $\mathrm{LiCl}-\mathrm{LiBr}-\mathrm{KBr}$ Eutectic and 25\% $\mathrm{MgO}$ at $75 \% \mathrm{TD}$

7 Deformation of Separator Pellets Based on LiCl-LiBr-KBr Eutectic and 30\% $\mathrm{MgO}$ at $75 \%$ TD.

8 Deformation of Separator Pellets Based on LiBr-KBr-LiF Eutectic and 25\% $\mathrm{MgO}$ at $75 \% \mathrm{TD}$

9 Deformation of Separator Pellets Based on $\mathrm{LiBr}-\mathrm{KBr}-\mathrm{LiF}$ Eutectic and 30\% $\mathrm{MgO}$ at $75 \% \mathrm{TD}$.

10 Deformation of Separator Pellets Based on LiCl-LiBr-LiF Electrolyte and $30 \% \mathrm{MgO}$ at $75 \% \mathrm{TD}$

11 Deformation of Separator Pellets Based on LiCl-LiBr-LiF Electrolyte and $35 \% \mathrm{MgO}$ at $75 \% \mathrm{TD}$

12 Signs of Coefficients for Variables in Polynomial Expressions for Deformation of EB Pellets

13 Optimum Densities for Various EBs Examined in This Work 



\section{Deformation Study of Separator Pellets for Thermal Batteries}

\section{Introduction}

Thermally activated batteries use a molten-salt electrolyte that serves as a separator between the anode [e.g., $\mathrm{Li}(\mathrm{Si})]$ and the cathode (e.g., $\mathrm{FeS}_{2}$ ) of each cell. When the battery is activated, a pyrotechnic heat source raises the temperature of each cell above the melting point of the electrolyte $\left(352^{\circ} \mathrm{C}\right.$ for the $\mathrm{LiCl}-\mathrm{KCl}$ eutectic) and allows current to be drawn from the battery.

The electrolyte is typically immobilized by using an inert oxide binder, such as $\mathrm{MgO}$ powder, that is thermodynamically stable to the $\mathrm{Li}$ and Li-alloy anodes used in the batteries. The electrolyte-binder (EB) mixes are prepared by blending together the $\mathrm{MgO}$ and the electrolyte powders and by fusing the material above the melting point of the electrolyte (typically, for up to $16 \mathrm{~h}$ ). After granulation, the EB powder is pressed into separator pellets. A cross- sectional representation of a typical thermal cell is shown in Figure 1.

The mechanical properties of the separator pellet are important for proper operation of thermal batteries. If there is excessive deformation (plastic flow) of the pellet when the electrolyte is molten, intracell shorting can occur with thin cells if the anode and cathode pellets come into contact. In addition, electrolyte leakage can occur, leading to intercell parasitic currents as the electrolyte dewets the $\mathrm{MgO}$ and wicks into the ceramic blanket wrap used to insulate the battery.

On the other hand, if there is insufficient deformation, unacceptable wetting of the anode and cathode pellets by the molten separator pellet can occur. This can lead to a high electrical resistance at the separator-anode and separator-cathode interfaces during discharge, and thus can adversely impact performance.

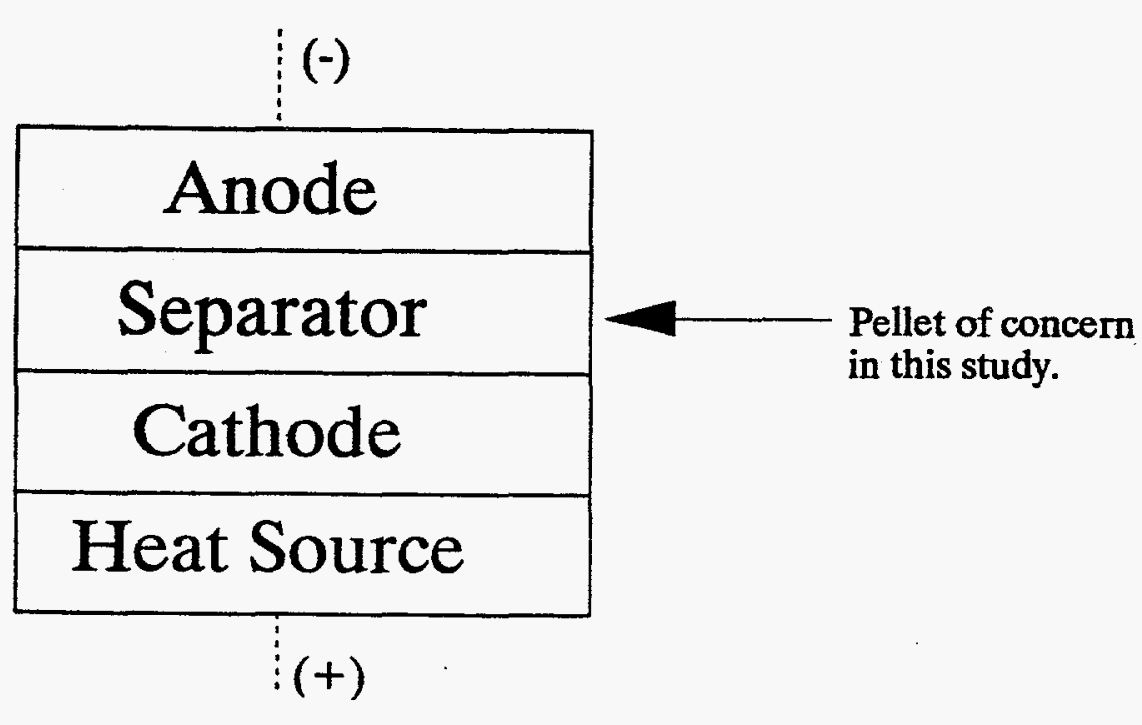

Figure 1. Cross-Sectional Representation of a Thermal Cell 
Factors that influence the deformation properties of the separator pellet include the $\mathrm{MgO}$ content, the applied (stack) pressure, the temperature, and the percent of theoretical density (porosity). In the past, an applied pressure of $14.3 \mathrm{psig}(99 \mathrm{kPa})$, a temperature of $530^{\circ} \mathrm{C}$, and a nominal density of $75 \%$ of theoretical were used as test levels for measuring the deformation of separator pellets. ${ }^{1}$ However, the relative sensitivity of the deformation to these test levels was unknown. Therefore, a study was undertaken to examine the effect of the above factors on the deformation properties of separator pellets made from EB mixes formulated with four different electrolytes:

- the standard $\mathrm{LiCl}-\mathrm{KCl}$ eutectic

- a low-melting $\mathrm{LiCl}-\mathrm{LiBr}-\mathrm{KBr}$ eutectic

- a low-melting LiBr-KBr-LiF eutectic

- an all-Li electrolyte (LiCl-LiBr-LiF minimum-melting composition).

For each electrolyte type, an experimental strategy was developed to facilitate an understanding of the individual and interactive effects of the various factors on deformation. Analysis of the resulting experimental data led to the development of response-surface plots that relate the deformation to the levels of the experimental factors.

\section{Experimental Procedures}

\section{Materials}

Electrolytes - Reagent-grade halide salts were used in the preparation of the various electrolytes. The compositions of the four electrolytes used and their melting points are summarized in Table 1.

The individual halide salts were vacuum dried at $100^{\circ} \mathrm{C}$ for $40 \mathrm{~h}$ before use. The appropriate amounts of the salts were blended for each electrolyte composition and the mixes were fused at $650^{\circ} \mathrm{C}$ for $3 \mathrm{~h}$. After quenching on an Inconel tray, the salts were pulverized to -325 mesh using a MikroPulverizer (Mikropul, Summit, NJ).

EB Mixes - The EB mixes were prepared by blending the electrolyte and $\mathrm{MgO}$ powders together using Freon TF®. ${ }^{4}$ Before blending, the $\mathrm{MgO}$ was first baked at $600^{\circ} \mathrm{C}$ for $4 \mathrm{~h}$ to decompose carbonates and hydroxides of $\mathrm{Mg}$. The EB mixes were heated in a $35^{\circ} \mathrm{C}$ convection oven to remove the Freon TF. Except for the all-Li EB mix, which was fused at $500^{\circ} \mathrm{C}$ for $16 \mathrm{~h}$, the EB mixes were then fused at $400^{\circ} \mathrm{C}$ for $16 \mathrm{~h}$. After fusion, the EB mixes were granulated to -60 mesh prior to pelletizing the separator discs to the desired densities. The theoretical densities (TDs) were calculated using the densities of the individual components, assuming addi-tivity of volumes. After pressing, the separator pellets were vacuum dried at $100^{\circ} \mathrm{C}$ for $16 \mathrm{~h}$.

All the separator pellets in this study were $3.28 \mathrm{~cm}(1.25 \mathrm{in})$ in diameter and $0.150 \mathrm{~cm}$ $(0.0591 \mathrm{in})$ thick. The masses of the pellets were adjusted to obtain the desired $\%$ TD for these dimensions.

All material processing and handling and all deformation testing were conducted in a dry room maintained at $<300 \mathrm{ppm}$ water vapor $(<1 \%$ relative humidity). 
Table 1. Electrolytes Evaluated in Separator Study

\begin{tabular}{llc} 
Name & Electrolyte Composition, w/o & ${ }^{*}$ Melting Point, ${ }^{\circ} \mathrm{C}$ \\
\hline Standard & $55 \mathrm{KCl} / 45 \mathrm{LiCl}$ & 352 \\
LM\#1 & $51.41 \mathrm{KBr} / 36.54 \mathrm{LiBr} / 12.05 \mathrm{LiCl}$ & 321 \\
$\mathrm{LM} 22$ & $57.33 \mathrm{LiBr} / 42 \mathrm{KBr} / 0.67 \mathrm{LiF}$ & $313^{*}$ \\
All-Li & $68.4 \mathrm{LiBr} / 22 \mathrm{LiCl} / 9.6 \mathrm{LiF}$ & 437
\end{tabular}

*As determined by differential scanning calorimetry in this laboratory
**LM designates "low melting."
'This electrolyte was reported as having a melting point of $280^{\circ} \mathrm{C}$. (See Reference 2). This
composition was subsequently found to be slightly off eutectic during subsequent work at Sandia
National Laboratories (SNL) and at Argonne National Laboratory (ANL). (See Reference 3.)

\section{Deformation Tester}

The experimental setup used for deformation testing of the separator pellets was identical to that used for characterizing catholyte pellets for $\mathrm{Ca} / \mathrm{CaCrO}{ }_{4}$ thermal batteries. ${ }^{5}$ Basically, the setup consisted of a single-cell tester fitted with a linear variable differential transformer (LVDT) to monitor the change in the thickness of the pellet during the test. The applied pressure to the separator pellet was changed by adjusting the mass of the weight attached to the ram of the tester.

\section{Statistical Experimental Design}

The test matrix used for this study is summarized in Table 2. Except for the $\% \mathrm{MgO}$, each of the experimental factors was tested at three levels, for $3^{3}$ or 27 experiments per each EB composition. Normally, the lowest nominal \% TD was 65 , although values as low as 55 were used in several instances. Three pellets were tested at each test condition and the mean deformation and standard deviation were determined.

The selected ranges of factor levels were based on previous studies and physical con- straints. The lower TD limit was determined by the fragility of the separator pellet. Because electrolyte leakage becomes excessive at TDs above $90 \%$, the upper limit was maintained below this level.

The temperatures chosen are those that span the typical operating range of a normally functioning thermal battery. The levels of $\mathrm{MgO}$ chosen for each electrolyte were determined in earlier empirical studies with these materials. ${ }^{6}$ The lower applied pressure is typical of those measured in stack-relaxation experiments with $\mathrm{Li}(\mathrm{Si}) / \mathrm{FeS}_{2}$ thermal batteries. The upper pressure limit was determined by the total mass that could be handled by the deformation tester for the size of the separator pellet used in the study.

\section{Statistical Analysis and Modeling} The percent reduction in separator pellet thickness was not modeled directly as a function of the experimental factors. Instead, the logit transformation of the percent reduction in thickness was modeled. The logit-transformed percent reduction in thickness is $\ln [\mathrm{Y} /(100-\mathrm{Y})]$, where $\mathrm{Y}$ is the mean percent reduction in thickness. In gen- 
Table 2. Experimental Factors and Associated Levels

Factor

$\%$ Theoretical Density (Nominal)

Temperature, ${ }^{\circ} \mathrm{C}$

Applied Pressure, psig (kPa)

$\% \mathrm{MgO}$

a. Standard. Electrolyte

b. LM\#1 Electrolyte

c. LM\#2 Electrolyte

d. All-Li Electrolyte
Levels

$65,75,85$

$500,530,560$

$14.4(99), 23.8(157), 32.8(226)$

35,40

30,35

25,30

30,35 eral, the models considered are subsets of the complete quadratic model:

$$
\begin{aligned}
& \ln [Y /(100-Y)]=b_{0}+b_{1} P+b_{2} T+b_{3} D \\
& +b_{12} P T+b_{13} P D+b_{23} T D+b_{11} P^{2} \\
& +b_{22} T^{2}+b_{33} D^{2}+e
\end{aligned}
$$

where:

$\mathbf{P}=$ applied pressure (psig)

$\mathrm{T}=$ temperature $\left({ }^{\circ} \mathrm{C}\right)$

$\mathrm{D}=\% \mathrm{TD}$

the $b_{i}$ 's and $b_{i j}$ 's are model parameters to be estimated and $e$ is a random error.

The $b_{i j}$ term relates to the magnitude of the interaction between the $i$ th and $j$ th factors; i.e., the combined effect of these factors that is in addition to the simple effect $b_{i}$ and $b_{j}$. (Pressure units of $p s i g$ were used for modeling purposes, rather than $\mathrm{kPa}$, because thermal-battery design engineers normally use these units.)
The purpose of the logit transformation was to make the errors $(e$ 's) symmetric and to equalize the variance of $e$ over the range of Y. For a given mixture, the model selected was the one with the fewest acceptable number of terms that fit the experimental data. Table 3 shows the model terms included for each mixture. All models included the $b_{0}$ term (intercept)

For example, for the EB mixtures with LM\#1 or LM\#2 electrolytes, the models selected were of the form:

$\ln [Y /(100-Y)]=b_{0}+b_{1} P+b_{2} T+$

$b_{3} D+b_{13} P D+b_{33} D^{2}+e$

The parameters associated with each model (e.g., $b_{0}, b_{1}, b_{2}, b_{3}, b_{13}$, and $b_{33}$ for the LM\#1 and $\mathrm{LM} \# 2$ electrolytes) were estimated by weighted least-squares regression. In order to avoid numerical problems during the computation of the parameter estimates (frequently present for polynomial regression analysis), the study factors were centered and scaled as follows: 
Table 3. Terms Used for Modeling Deformation Data

Electrolyte

$\% \mathrm{MgO}$ in EB

Model Terms Included

LiCl-KCl Electrolyte

35

LiCl-KCl Electrolyte

40

$\mathbf{P}$

$\mathbf{P} \quad \mathrm{D}$

LM\#1 Electrolyte

25

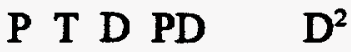

LM\#1 Electrolyte

30

P T D PD $\quad D^{2}$

LM\#2 Electrolyte

25

$\begin{array}{lll}P & \text { T D PD } \quad D^{2}\end{array}$

LM\#2 Electrolyte

30

P T D PD $\quad \mathrm{D}^{2}$

All-Li Electrolyte

$P$
$P$

All-Li Electrolyte

$P$ T D PD $P^{2} D^{2}$

$\mathrm{T}^{*}=(\mathrm{T}-530) / 30, \mathrm{P}^{*}=(\mathrm{P}-23.8) / 10$,

and $D^{*}=(D-70) / 20$.

Weighted least-squares regression gives weight to each measurement triplet that depends on the mean and standard deviation of the three measurements. The weights $\left(w_{i}^{\prime} s\right)$ associated with the ith experimental conditions are defined as

$\mathrm{w}_{\mathrm{i}}=[\mathrm{Y}(100-\mathrm{Y})]^{2}$ for $\mathrm{S}<1$

or $w_{i}=[Y(100-Y) / S]^{2}$ for $S>=1$.

where $Y$ and $S$ are the average and standard deviation, respectively, of the three measurements of the percent reduction in thickness.

For a fixed standard deviation, $\mathrm{S}$, the weight is maximized when $\mathrm{Y}=50 \%$.

The data from the deformation tests were analyzed using PROC Reg in SAS.'

DISSPLA (on a VAX platform) was used to produce the contour plots that appear in this report. ${ }^{8}$ Currently, a number of software packages for contour plotting are now available for use with a PC. These include Axum (TriMetrix, Seattle, WA), Surfer (Golden Software, Golden, CO), Design Expert (Stat-Ease,Inc., Minneapolis, MN), TechPlot (Polysoft, Salt Lake City, UT), and Minitab (Minitab, Inc., State College, PA).

\section{Results}

\section{LiCl-KCl (Standard) Electrolyte: $35 \% \mathrm{MgO}$}

A typical deformation profile for a separator pellet formulated with $\mathrm{LiCl}-\mathrm{KCl}$ eutectic and $35 \% \mathrm{MgO}$ and pressed to $75 \% \mathrm{TD}$ is shown in Figure 2 for a temperature of $530^{\circ} \mathrm{C}$ and an applied pressure of $14.4 \mathrm{psig}(99 \mathrm{kPa})$.

The change in pellet thickness (slumping) was almost instantaneous once the electrolyte melted. (This behavior was found to be uniform for all the EB mixes evaluated, regardless of the electrolyte composition.) For the purpose of this study, the reduction in thickness at 3 min elapsed time was taken as the measure of deformation. The deformation data for separator pellets based on the 


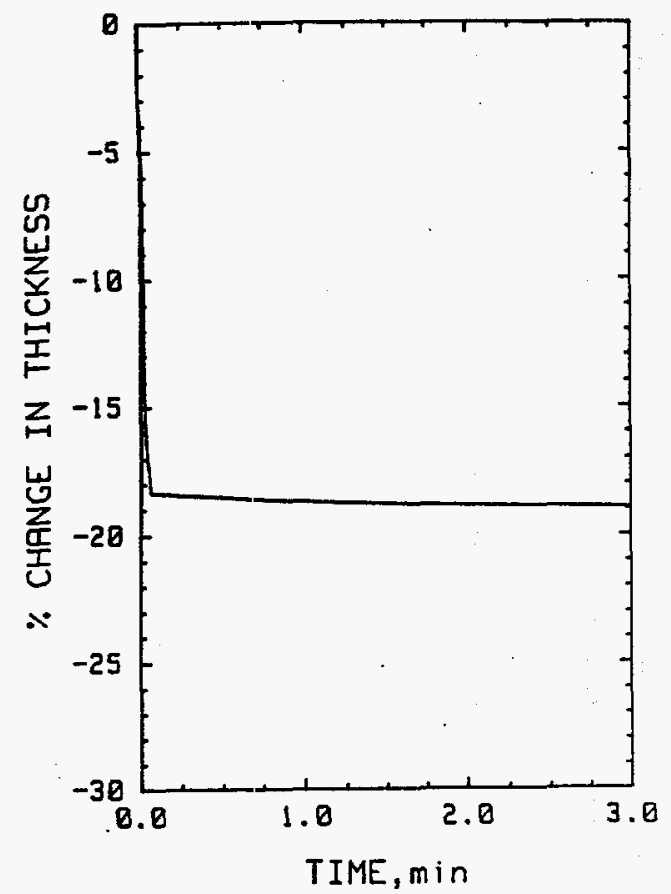

Figure 2. Representative Deformation Profile at $530^{\circ} \mathrm{C}$ and 14.3 Psig Applied Pressure of Separator Pellet Based on LiCl-KCl Eutectic and 35\% MgO)

$\mathrm{LiCl}-\mathrm{KCl}$ eutertic are summarized in Appendix A-1. [Several tests were conducted at $28.2 \mathrm{psig}(194 \mathrm{kPa})$ applied pressure to better define the system curvature and to enable modeling for this EB composition.]

Modeling. The mean values and standard deviations for separator pellet deformation were analyzed by a least-squares regression analysis. The polynomial coefficients (parameter estimates) for the EB mix based on $\mathrm{LiCl}-\mathrm{KCl}$ eutectic and $35 \% \mathrm{MgO}$ are listed in Appendix B-1. Appendix C-1 lists the percentage reduction in thickness (deformation) predicted by the model and the corresponding measured values for the various experimental conditions. The estimated values of deformation were calculated from:

$Y=100\left[\left(e^{z} /\left(1+e^{z}\right)\right]\right.$ where $\mathrm{z}$ is the logit-model expression evaluated at the appropriate values of $P^{*}, T^{*}$, and $D^{*}$, with parameter estimates (e.g., $b_{0}$ ) substituted for the corresponding model parameter. For the $\mathrm{EB}$ with $\mathrm{LiCl}-\mathrm{KCl}$ eutectic and $35 \% \mathrm{MgO}$, the expression for $\mathrm{z}$ is:

$$
\begin{aligned}
& z=b_{0}+b_{1} P^{*}+b_{3} D^{*}+b_{13} P^{*} D^{*}+b_{11} P^{2} \\
& +b_{33} D^{2}+\gamma P^{2} D^{2}
\end{aligned}
$$

For example, at $80 \%$ TD and an applied pressure of $23.8 \mathrm{psig}, \mathrm{D}^{*}$ and $\mathrm{P}^{*}$ are 0.280 and 0.90 , respectively. Using these values in Eq. $6, \mathrm{z}$ is calculated to be 0.106 and, using $\mathrm{Eq} .5, \mathrm{Y}$ is found to be $52.6 \%$. The values of $Y$ in Appendix C-1 were determined in this way.

The fraction of the variation of the logittransformed response about the mean value that is explained by the model can be summarized by the squared correlation coefficient, $\mathbf{R}^{2}$. In this case, $\mathbf{R}^{2}=0.93$. Thus, $93 \%$ of the variation of the response was explained by the model.

The estimated response surfaces (contour plots) for deformation are shown in Figure 3 as a function of $\%$ TD and applied pressure for temperatures of $500^{\circ}$ to $560^{\circ} \mathrm{C}$. (Because of the lack of temperature dependence, only a single graph is presented.)

The arrow on the figure refers to the nominal value called out for the density of separator pellets for $\mathrm{Li}(\mathrm{Si}) / \mathrm{FeS}_{2}$ thermal batteries designed at SNL. The data of Figure 3 indicate that the design point should be moved to $73.4 \% \mathrm{TD}$, to minimize the effect of applied pressure on pellet deformation.

Empirically, the amount of separator pellet deformation that is acceptable for battery purposes lies within the range of $\sim 15 \%$ to 


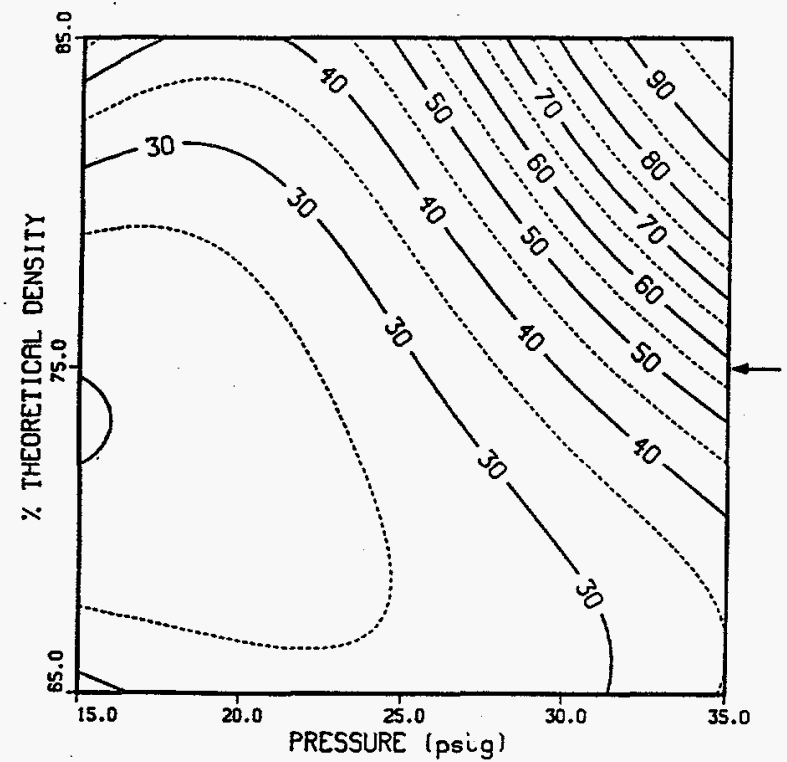

Figure 3. Contour Plot for Estimated Deformation of EB Pellets Based on $\mathrm{LiCl}-\mathrm{KCl}$ Eutectic and 35\% $\mathrm{MgO}$ as a Function of Applied Pressure and \% Theoretical Density for Temperature of $500^{\circ} \mathrm{C}$ to $560^{\circ} \mathrm{C}$

$30 \%$. Separator pellets with deformation higher than this will still work, but at the risk of reduced battery reliability if excessive electrolyte leakage into the battery insulation (wrap) occurs. Separator pellets with values lower than this range can experience reduced wetting of the adjacent anode and cathode pellets. The reduction in wetting can then increase the impedance of the cell, which can adversely impact performance.

Under a stack pressure of 15 psig, the deformation of separator pellets will be $-20 \%$, which is acceptable. (It should be emphasized here that the stack pressure refers to the pressure on the stack after the battery has been activated and does not represent the closing pressure, which is typically $>175$ psig.) However, once a stack pressure of $\sim 26$ psig is exceeded, deformation will be greater than $30 \%$. Under these conditions, potential problems could arise in a thermal battery during discharge, due to excessive separator pellet deformation.

The predicted deformation of separator pellets with $35 \% \mathrm{MgO}$ at an applied pressure of 15 psig and 35 psig is shown in Table 4 at the design point of $75 \%$ TD.

The deformation at an applied pressure of 35 psig is excessive; this could lead to problems during discharge for a thermal battery using such a material for the separator if the stack pressure is sustained at this level.

Thus, if information is available for the critical parameters (namely, applied pressure, $\%$ $\mathrm{TD}$, and temperature) during discharge of a thermal battery, such contour maps are valuable from a thermal-battery design perspective. Because pellets are pressed to a specific density, the \% TD will already be known. Stack pressure can be recorded by incorporating a load cell into a battery. [Such tests have already been conducted at SNL and will be the subject of a separate report. ${ }^{8}$ ] Stack temperatures can be recorded by insertion of a thin, flattened thermocouple into the battery stack during construction. Care must be taken, however, to avoid possible shorting of cells-especially with thin cells, where pellet thickness can be as low as $0.020 \mathrm{~cm}$ (0.008 in).

\section{LiCl-KCl (Standard) Electrolyte: 40\% MgO}

Modeling. The polynomial coefficients for the deformation model for the EB with $40 \%$ $\mathrm{MgO}$ are listed in Appendix B-2. The percentage reduction in thickness estimated by the model is listed in Appendix C-2, with the corresponding measured values for the range of experimental conditions. Note that this EB composition uses the simplest model for deformation of all the mixes examined in this 


\section{Table 4. Deformation of Separator Pellets Based on $\mathrm{LiCl}-\mathrm{KCl}$ Eutectic and $35 \% \mathrm{MgO}$ at $75 \% \mathrm{TD}^{*}$}

\begin{tabular}{ccc} 
Temperature, ${ }^{\circ} \mathrm{C}$ & Pressure, psig & \% Reduction in Thickness \\
\hline & & \\
$500-560$ & 15 & 20.1 \\
$500-560$ & 35 & 58.2
\end{tabular}

* Predicted using the model shown Appendix B-1.

study. It includes only terms for \% TD and applied pressure and has no interaction terms (see Table 3).

One area where poor agreement was observed between predicted and measured values of deformation is at the highest pressure and \% TD. Deformation between 55\% and $60 \%$ was measured, while the model predicts only $13 \%$. The squared correlation coefficient $\left(\mathbf{R}^{2}\right)$ was 0.97 . Thus, $97 \%$ of the variation of the logit-transformed response was explained by the model. (The highpressure, high-\% TD deformation data were not included in the regression analysis or computation of $\mathbf{R}^{2}$.)

The estimated response surface for deformation is shown in Figure 4 as a function of $\% \mathrm{TD}$ and applied pressure for temperatures of $500^{\circ} \mathrm{C}$ to $560^{\circ} \mathrm{C}$. (The upper right-hand corner of the contour plot is blanked out because of the poor agreement of the model with the experimental data in this region.) The predicted deformation of separator pellets with $40 \% \mathrm{MgO}$ at an applied pressure of 15 psig and 35 psig is shown in Table 5 at the design poirt of $75 \% \mathrm{TD}$.

The deformation is well within the acceptable response for the entire pressure region.
In comparison, the deformation for the comparable EB with $35 \% \mathrm{MgO}$ while acceptable at 15 psig $(20.1 \%)$ was high for the highest pressure $(58.2 \%)$ (Table 4 ). Thus, the deformation of separator pellets of $\mathrm{LiCl}-\mathrm{KCl}$ eutectic with $40 \% \mathrm{MgO}$ is much more "robust" to changes in applied pressure and \% TD. The specified design point of 75\% TD appears acceptable for this EB composition.

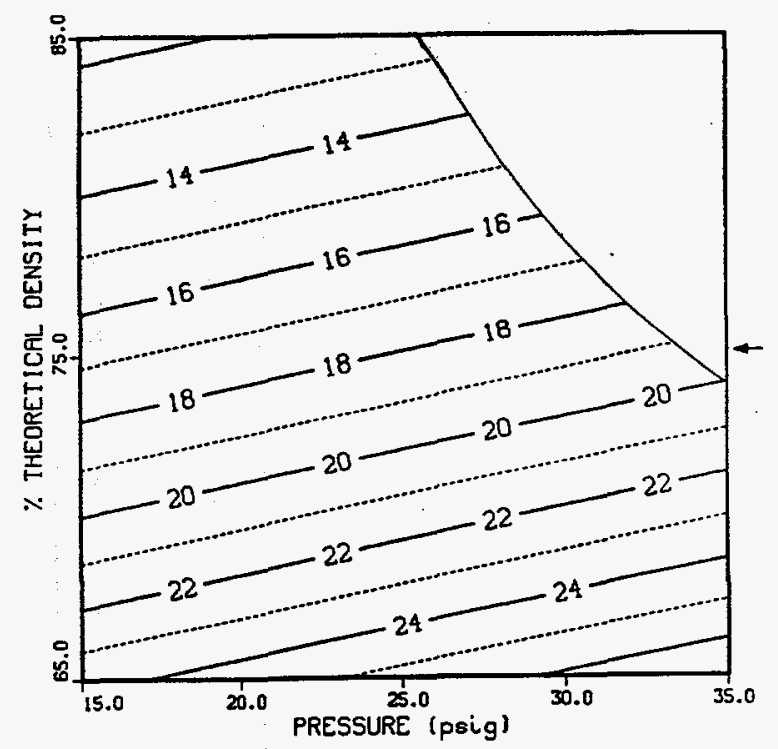

Figure 4. Contour Plot for Estimated Deformation of EB Pellets Based on $\mathrm{LiCl}-\mathrm{KCl}$ Eutectic and $40 \%$ $\mathrm{MgO}$ as a Function of Applied Pressure and \% Theoretical Density for Temperatures of $500^{\circ} \mathrm{C}$ $560^{\circ} \mathrm{C}$ 
Table 5. Deformation of Separator Pellets Based on LiCl-KCl Eutectic and $40 \% \mathrm{MgO}$ at $75 \% \mathrm{TD}^{*}$

Temperature, ${ }^{\circ} \mathrm{C}$

Pressure, psig

$\%$ Reduction in Thickness

$500-560$

15

16.8

$500-560$

*Predicted using the model shown in Appendix B-2.

\section{LiCl-LiBr-KBr (LM\#1) Electrolyte: 25\% MgO}

The deformation data for separator pellets based on the LiCl-LiBr-KBr eutectic electrolyte and $25 \% \mathrm{MgO}$ are summarized in Appendix A-2

Modeling. The polynomial coefficients for the deformation model for the LM\#1 EB with $25 \% \mathrm{MgO}$ are listed in Appendix B-3. The percentage reduction in thickness predicted by the model is listed in Appendix $\mathrm{C}-3$, with the corresponding measured values for the various experimental conditions.

Note that the model for this EB mix includes temperature, pressure, and density terms as well as a squared term (D) and an interaction term (PD). In this case, $\mathbf{R}^{2}$ was 0.98 .

The estimated response surfaces for deformation are shown in Figures $5 \mathrm{a}$ and $5 \mathrm{~b}$ as a function of \% TD and applied pressure for temperatures of $500^{\circ} \mathrm{C}$ and $560^{\circ} \mathrm{C}$, respectively. The predicted deformation of separator pellets with $25 \% \mathrm{MgO}$ at applied pressures of 15 psig and $35 \mathrm{psig}$ is shown in Table 6 at the design point of $75 \%$ TD for temperatures of $500^{\circ} \mathrm{C}$ and $560^{\circ} \mathrm{C}$.

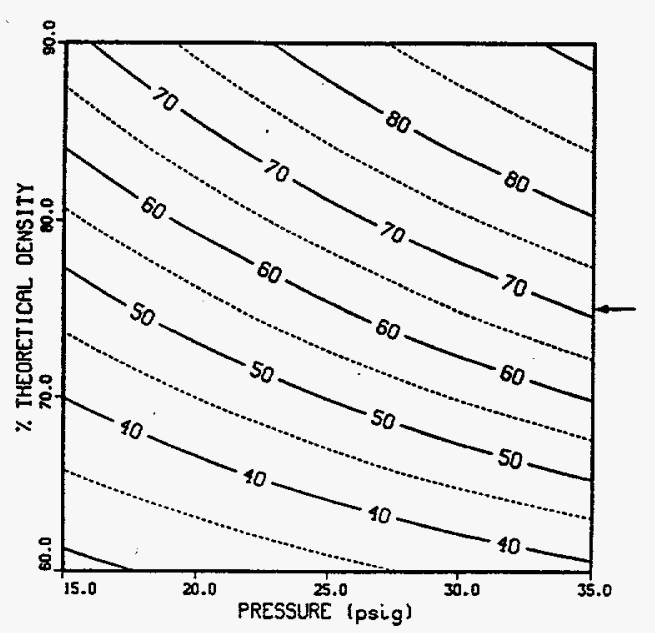

Figure 5A. Contour Plot for Estimated Deformation of EB Pellets Based on LiCl- $\mathrm{LiBr}-\mathrm{KBr}$ Eutectic and $25 \% \mathrm{MgO}$ as a Function of Applied Pressure and \% Theoretical Density for $500^{\circ} \mathrm{C}$

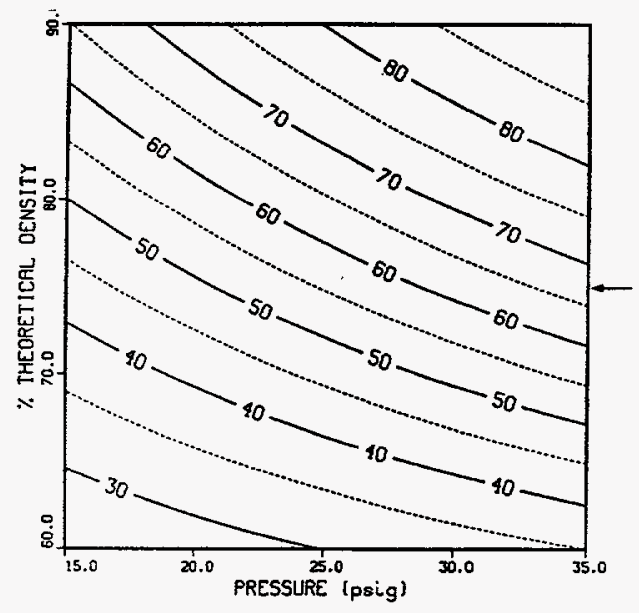

Figure 5B. Contour Plot for Estimated Deformation of EB Pellets Based on $\mathrm{LiCl}-\mathrm{LiBr}-\mathrm{KBr}$ Eutectic and 25\% MgO as a Function of Applied Pressure and \% Theoretical Density for $560^{\circ} \mathrm{C}$ 
Table 6. Deformation of Separator Pellets Based on LiCl-LiBr-KBr Eutectic and $25 \% \mathrm{MgO}$ at $75 \% \mathrm{TD}^{*}$

Temperature, ${ }^{\circ} \mathrm{C} \quad$ Pressure, psig $\quad \%$ Reduction in Thickness

$\begin{array}{lll}500 & 15 & 46.8 \\ 500 & 35 & 70.8 \\ 560 & 15 & 42.8 \\ 560 & 35 & 67.3\end{array}$

*Predicted using the model shown in Appendix B-3

Pellet deformation was excessive ( $>30 \%)$ in all cases. As a result, this EB composition would not be acceptable for use in separator pellets in thernal batteries at the design point of $75 \% \mathrm{TD}$. Lowering the pellet density to $60 \%$ TD would not solve the problem

\section{LiCl-LiBr-KBr (LM\#1) Electrolyte: $30 \% \mathrm{MgO}$}

The deformation data for separator pellets based on the $\mathrm{LiCl}-\mathrm{LiBr}-\mathrm{KBr}$ eutectic electrolyte and 30\% MgO are summarized in Appendix C-2. Increasing the binder content from $25 \%$ to $30 \%$ reduced the separatorpellet deformation considerably.

Modeling. The polynomial coefficients for the deformation model for the LM\#1 electrolyte with $30 \% \mathrm{MgO}$ are listed in Appendix B-4. The model for this EB composition is the same as for the EB with $25 \% \mathrm{MgO}$. The percent reduction in thickness predicted by the model is listed in Appendix C-3 with the corresponding measured values for the various experimental conditions $\left(R^{2}=0.96\right)$.

The estimated response surfaces for pellet deformation are shown in Figures $6 \mathrm{a}$ and $6 \mathrm{~b}$ for temperatures of $500^{\circ} \mathrm{C}$ and $560^{\circ} \mathrm{C}$, re- spectively. The wide contours between $60 \%$ and $85 \%$ TD are highly desirable because this indicates that deformation is relatively insensitive to large changes in applied pressures and $\% \mathrm{TD}$. The estimated deformation of separator pellets with $30 \% \mathrm{MgO}$ at applied pressures of $15 \mathrm{psig}$ and $35 \mathrm{psig}$ is shown in Table 7 at the pellet-density design point of $75 \%$ TD for temperatures of $500^{\circ} \mathrm{C}$ and $560^{\circ} \mathrm{C}$.

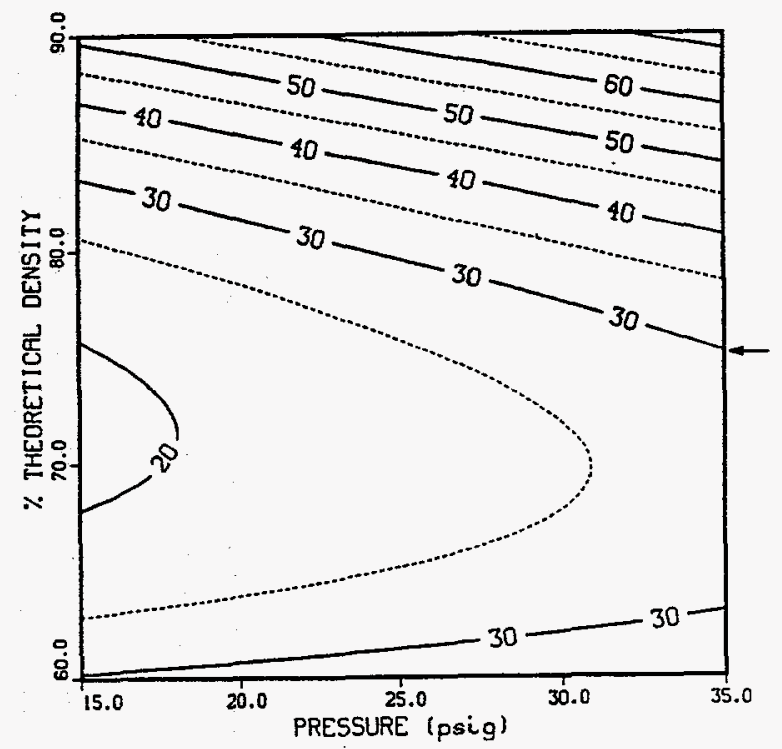

Figure 6A. Contour Plot for Estimated Deformation of EB Pellets Based on LiCl-LiBr-KBr Eutectic and $30 \% \mathrm{MgO}$ as a Function of Applied Pressure and \% Theoretical Density for $500^{\circ} \mathrm{C}$. 
Table 7. Deformation of Separator Pellets Based on LiCl-LiBr-KBr Eutectic and $30 \% \mathrm{MgO}$ at $75 \% \mathrm{TD}^{*}$

\begin{tabular}{ccc} 
Temperature, ${ }^{\circ} \mathrm{C}$ & Pressure, psig & \% Reduction in Thickness \\
\hline 500 & 15 & 19.6 \\
500 & 35 & 29.9 \\
560 & 15 & 18.1 \\
560 & 35 & 27.8
\end{tabular}

*Predicted using the model shown in Appendix B-3

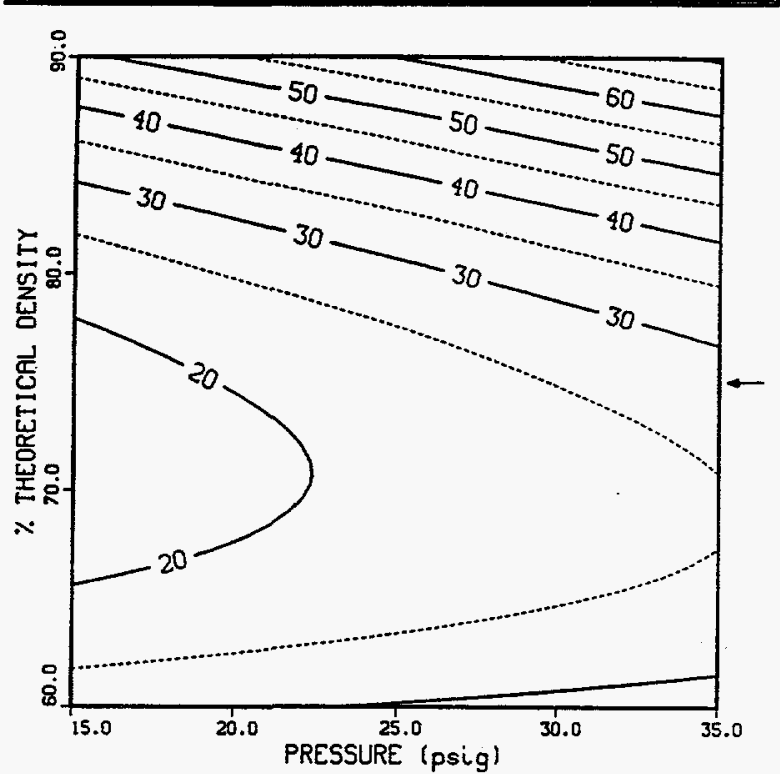

Figure 6B. Contour Plot for Estimated Deformation of EB Pellets Based on $\mathrm{LiCl}-\mathrm{LiBr}-\mathrm{KBr}$ Eutectic and $30 \% \mathrm{MgO}$ as a Function of Applied Pressure and \% Theoretical Density for $560^{\circ} \mathrm{C}$.

In all cases, the deformation lies within the acceptable region of $15 \%$ to $30 \%$. This EB composition is thus considered robust with respect to deformation over the full range of experimental conditions tested and would be a suitable candidate for use as a separator in thermal batteries. This robustness would be further enhanced if the pellet-density design point were reduced slightly from $75 \%$ to about $70 \%$ TD.

\section{LiBr-KBr-LiF (LM\#2) Electrolyte: $25 \%$ MgO}

The deformation data for separator pellets based on the $\mathrm{LiBr}-\mathrm{KBr}-\mathrm{LiF}$ eutectic electrolyte and $25 \% \mathrm{MgO}$ are summarized in Appendix A-3.

Modeling. The polynomial coefficients for the deformation model for the LM\#2 EB with $25 \% \mathrm{MgO}$ are listed in Appendix B-5. The percentage reduction in thickness predicted by the model is listed in Appendix $\mathrm{C}-5$, with the corresponding measured values for the various experimental conditions. The model for this EB mix is identical to that for the $L M \# 1$ EB mixes $\left(R^{2}=0.98\right)$.

The estimated response surfaces for pellet deformation are shown in Figures $7 \mathrm{a}$ and $7 \mathrm{~b}$ as a function of $\% \mathrm{TD}$ and applied pressure for temperatures of $500^{\circ} \mathrm{C}$ and $560^{\circ} \mathrm{C}$, respectively. The estimated deformation of separator pellets with $25 \% \mathrm{MgO}$ at applied pressures of 15 psig and 35 psig is listed in Table 8 at the pellet-density design point of $75 \% \mathrm{TD}$ for temperatures of $500^{\circ} \mathrm{C}$ and $560^{\circ} \mathrm{C}$. 


\section{Table 8. Deformation of Separator Pellets Based Upon LiBr-KBr-LiF}

Eutectic and $25 \% \mathrm{MgO}$ at $75 \% \mathrm{TD}^{*}$

\begin{tabular}{ccc} 
Temperature, ${ }^{\circ} \mathrm{C}$ & Pressure, psig & \% Reduction in Thickness \\
\hline 500 & 15 & 13.8 \\
500 & 35 & 25.4 \\
560 & 15 & 12.4 \\
560 & 35 & 23.2
\end{tabular}

\section{* Predicted using the model shown in Appendix B-5}

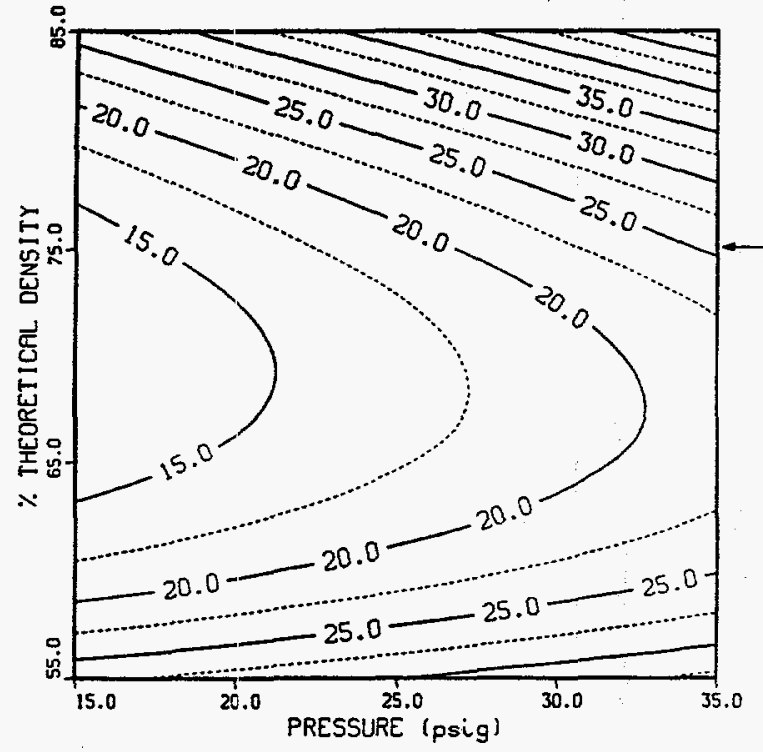

Figure 7A. Contour Plot for Estimated Deformation of EB Pellets Based on LiBr-KBr-LiF Eutectic and $25 \% \mathrm{MgO}$ as a Function of Applied Pressure and \% Theoretical Density for $500^{\circ} \mathrm{C}$

Pellet deformation was acceptable at the higher applied pressures and was only slightly below the nominal $15 \%$ deformation limit considered acceptable. Overall, this EB composition would be considered acceptable for use in separator pellets in thermal batteries.

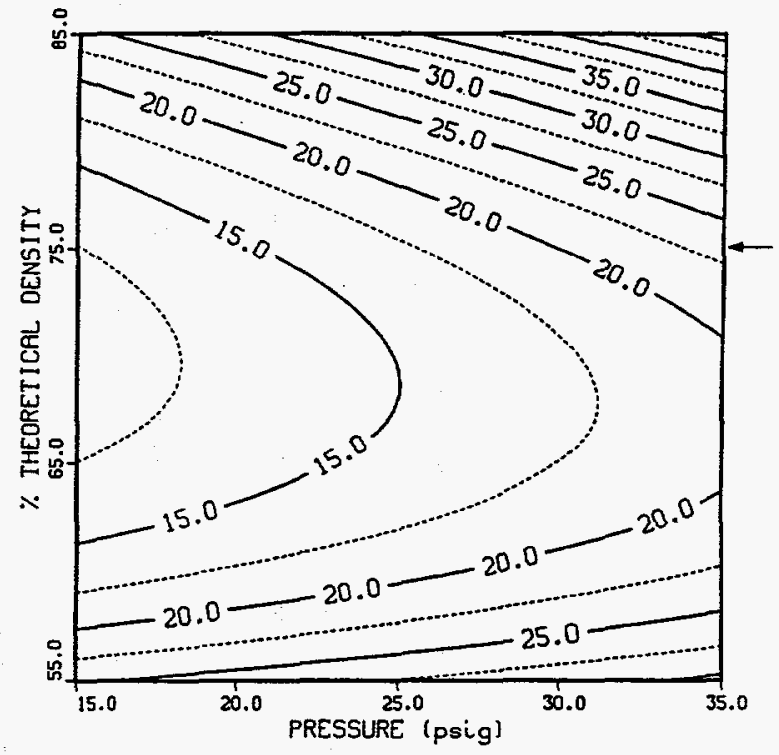

Figure 7B. Contour Plot for Estimated Deformat I of EB Pellets Based on LiBr-KBr-LiF Eutectic and $25 \% \mathrm{MgO}$ as a Function of Applied Pressure and \% Theoretical Density for $560^{\circ} \mathrm{C}$

The robustness of this EB composition to deformation would be enhanced if the pelletdensity design point were reduced from $75 \%$ to about $70 \%$ TD. 


\section{LiBr-KBr-LiF (LM\#2) Electrolyte: $\mathbf{3 0} \%$ MgO}

The deformation data for separator pellets based on the LiBr-KBr-LiF eutectic electrolyte and $30 \% \mathrm{MgO}$ are summarized in Appendix A-5. Increasing the binder content from $25 \%$ to $30 \%$ caused a large reduction in pellet deformation-especially at the higher pellet densities.

Modeling. The polynomial coefficients for the deformation model for the LM\#2 EB with 30\% MgO are listed in Appendix B-6. The percentage reduction in thickness predicted by the model is listed in Appendix C-6, with the corresponding measured values for the various experimental conditions. The model for this EB is identical to that for the $\mathrm{LM} \# 2$ EB with $25 \% \mathrm{MgO}\left(\mathrm{R}^{2}=0.92\right)$.

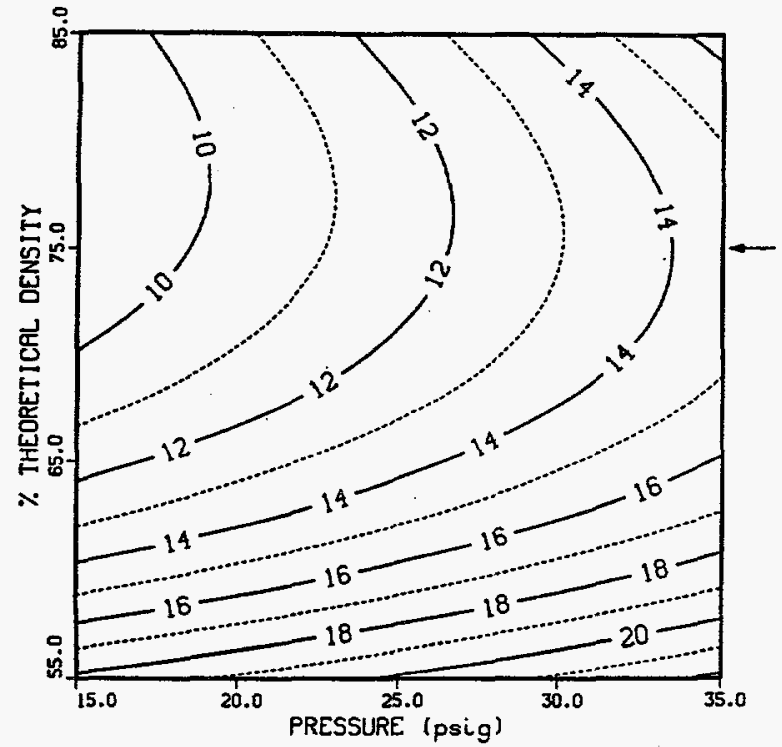

Figure 8A. Contour Plot for Estimated Deformation of EB Pellets Based on Li-Br-KBr-LiF Eutectic and $30 \% \mathrm{MgO}$ as a Function of Applied Pressure and \% Theoretical Density for $500^{\circ} \mathrm{C}$
The estimated response surfaces for pellet deformation are shown in Figures $8 \mathrm{a}$ and $8 \mathrm{~b}$ for temperatures of $500^{\circ} \mathrm{C}$ and $560^{\circ} \mathrm{C}$, respectively. The estimated deformation of separator pellets with $30 \% \mathrm{MgO}$ is listed in Table 9 at the pellet-density design point of $75 \% \mathrm{TD}$ for temperatures of $500^{\circ} \mathrm{C}$ and $560^{\circ} \mathrm{C}$.

Increasing the binder content of the LM\#2 EB from $25 \%$ to $30 \% \mathrm{MgO}$ caused a large reduction in deformation at the pellet-density design point of $75 \% \mathrm{TD}$. In all cases, the deformation was less than the lower limit of $15 \%$ considered acceptable for thermal-battery use. The data for the LM\#2 EB with $25 \% \mathrm{MgO}$ (Table 6) show this material to be preferred over the counterpart with $30 \%$ $\mathrm{MgO}$ (Table 7).

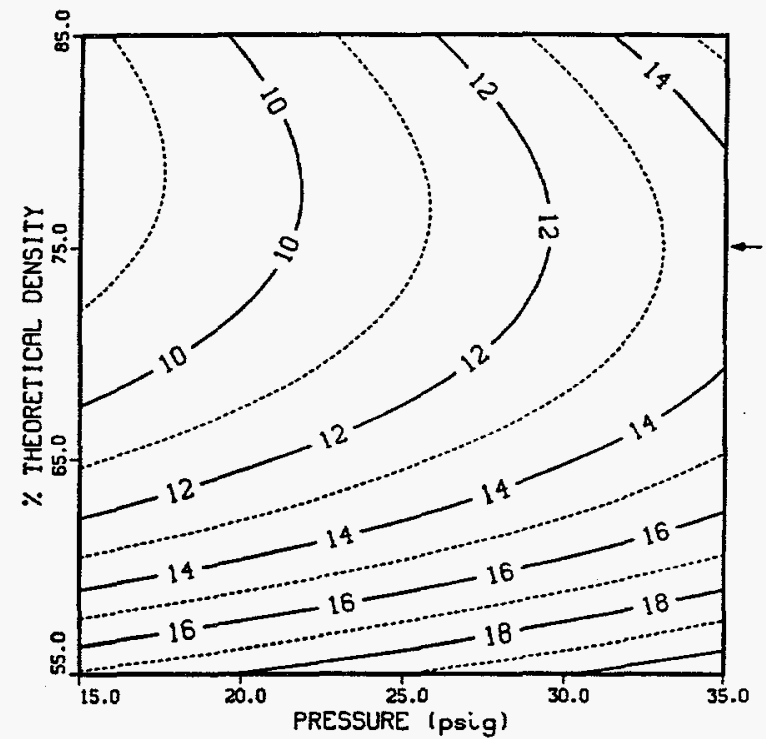

Figure 8B Contour Plot for Estimated Deformation of EB Pellets Based on Li-Br-KBr-LiF Eutectic and $30 \% \mathrm{MgO}$ as a Function of Applied Pressure and \% Theoretical Density for $560^{\circ} \mathrm{C}$ 
Table 9. Deformation of Separator Pellets Based on LiBr-KBr-LiF Eutectic and $30 \% \mathrm{MgO}$ at $75 \% \mathrm{TD}^{*}$

Temperature, ${ }^{\circ} \mathrm{C}$

Pressure, psig

$\%$ Reduction in Thickness

500

15

9.2

500

35

14.5

560

15

8.6

560

35

13.6

* Predicted using the model shown in Appendix B-5

\section{LiCl-LiBr-LiF (All-Li) Electrolyte: $30 \%$ MgO}

The deformation data for separator pellets based on the LiCl-LiBr-LiF minimum-melting electrolyte and $30 \% \mathrm{MgO}$ are summarized in Appendix A-4.

Modeling. The polynomial coefficients for the deformation model for the all-Li EB with $30 \% \mathrm{MgO}$ are listed in Appendix B-7. The percentage reduction in thickness predicted by the model is listed in Appendix C-7, with the corresponcling measured values for the various experimental conditions. The model is similar to those for the LM\#1 and LM\#2 EBs, except that it also includes a P-squared term $\left(\mathbf{R}^{2}=0.98\right)$.

The estimated response surfaces for pellet deformation are shown in Figures $9 a$ and $9 b$ for temperatures of $500^{\circ} \mathrm{C}$ and $560^{\circ} \mathrm{C}$, respectively. The estimated deformation of separator pellets at applied pressures of 15 psig and 35 psig is listed in Table 10 at the pellet-density design point of $75 \%$ TD for temperatures of $500^{\circ} \mathrm{C}$ and $560^{\circ} \mathrm{C}$.
Reducing the pellet-density design point from $75 \%$ TD to the optimum value of $70.5 \%$ TD would center the contour lines between 15 psig and 35 psig. However, the pellet deformation would still be $>\mathbf{3 0} \%$ for all conditions, which makes this EB composition unacceptable for use in separator pellets in thermal batteries.

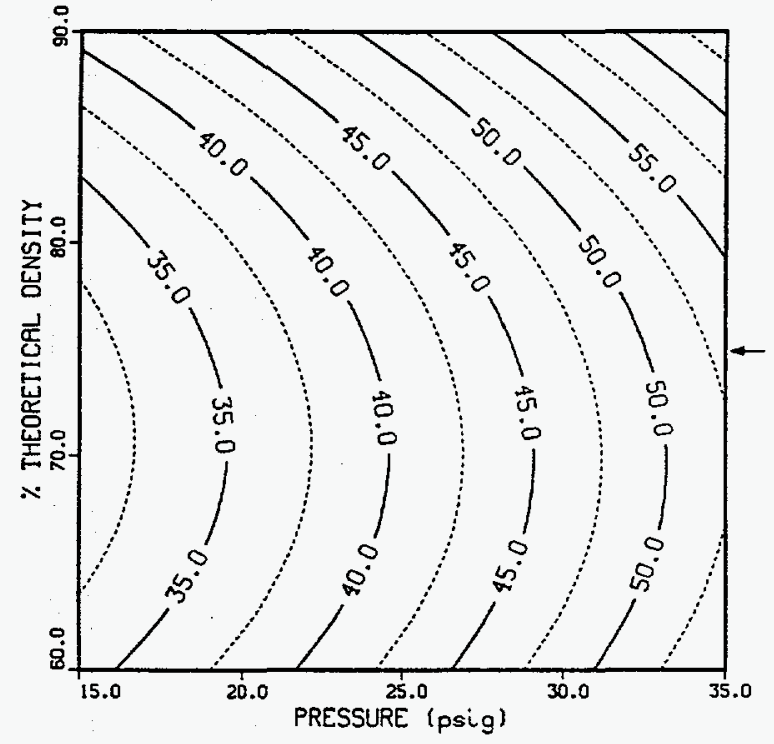

Figure 9A. Contour Plot for Estimated Deformation of EB Pellets Based on LiCl-LiBr-LiF Electrolyte and $30 \% \mathrm{MgO}$ as a Function of Applied Pressure and $\%$ Theoretical Density for $500^{\circ} \mathrm{C}$ 
Table 10. Deformation of Separator Pellets Based on LiCl-LiBr-LiF

Electrolyte and $30 \% \mathrm{MgO}$ at $75 \% \mathrm{TD}^{*}$

\begin{tabular}{ccc} 
Temperature, ${ }^{\circ} \mathrm{C}$ & Pressure, psig & \% Reduction in Thickness \\
\hline & & \\
500 & 15 & 35.3 \\
500 & 35 & 64.4 \\
560 & 15 & 32.3 \\
560 & 35 & 61.2
\end{tabular}

*Predicted using the model shown in Appendix B-7

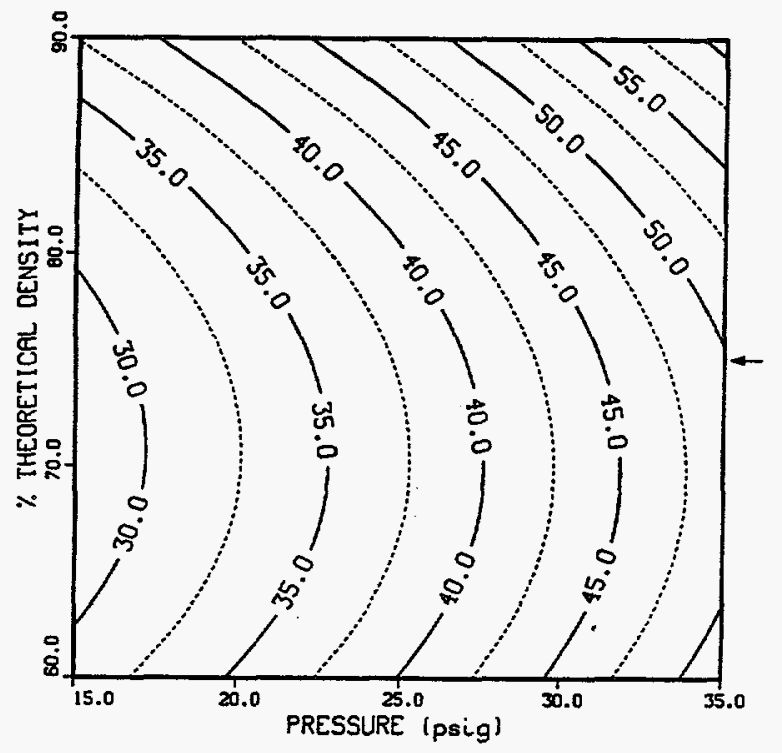

Figure 9B. Contour Plot for Estimated Deformation of EB Pellets Based on LiCl-LiBr-LiF Electrolyte and $30 \% \mathrm{MgO}$ as a Function of Applied Pressure and $\%$ Theoretical Density for $560^{\circ} \mathrm{C}$

\section{LiCl-LiBr-LiF (All-Li) Electrolyte: $35 \%$ MgO}

The deformation data for separator pellets based on the LiCl-LiBr-LiF minimum-melt- ing electrolyte and $35 \% \mathrm{MgO}$ are summarized in Appendix A-4. As expected, increasing the binder content from $30 \%$ to $35 \%$ resulted in a large reduction in the pellet deformation.

Modeling. The polynomial coefficients for the deformation model for the all-Li EB with $35 \% \mathrm{MgO}$ are listed in Appendix B-8. The percentage reduction in thickness predicted by the model is listed in Appendix C-8, with the corresponding measured values for the various experimental conditions. The model form is identical to that for the all-Li EB with $30 \% \mathrm{MgO}\left(\mathrm{R}^{2}=0.98\right)$.

The estimated response surfaces for pellet deformation are shown in Figures 10a and $10 \mathrm{~b}$ for temperatures of $500^{\circ} \mathrm{C}$ and $560^{\circ} \mathrm{C}$, respectively. The estimated deformation of separator pellets at applied pressures of 15 psig and 35 psig is listed in Table 11 at the pellet-density design point of $75 \%$ TD for temperatures of $500^{\circ} \mathrm{C}$ and $560^{\circ} \mathrm{C}$. 
Table 11. Deformation of Separator Pellets Based on LiCl-LiBr-LiF

Electrolyte and $35 \% \mathrm{MgO}$ at $75 \% \mathrm{TD}^{*}$

Temperature, ${ }^{\circ} \mathrm{C}$

Pressure, psig

$\%$ Reduction in Thickness

500

15

500

35

17.4

560

15

41.4

560

35

15.9

38.9

*Estimated using the model shown in Appendix B-7

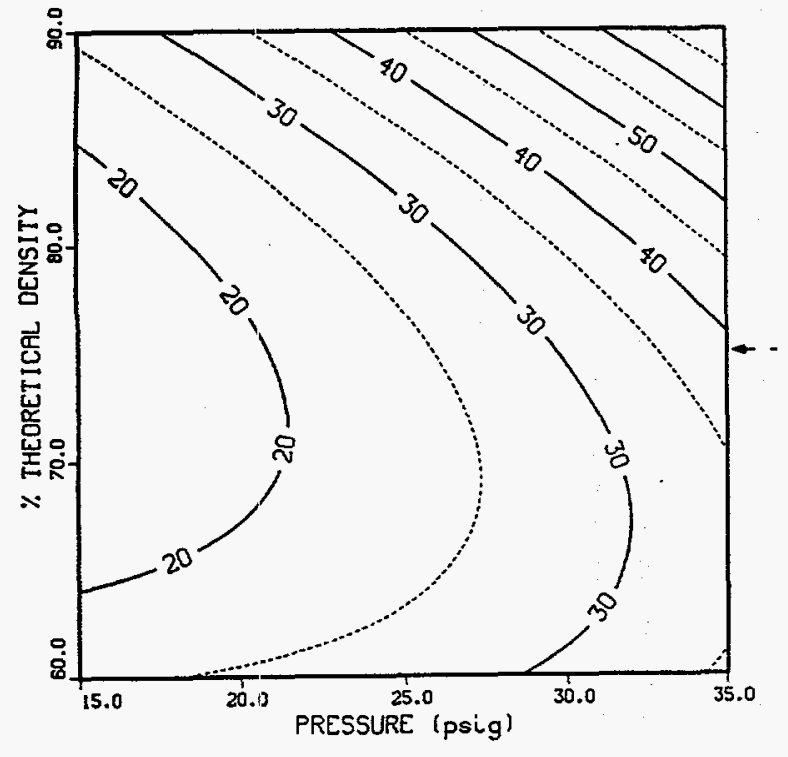

Figure 10A. Contour Plot for Estimated

Deformation of EB Pellets Based on LiCl-LiBr-LiF Electrolyte and $35 \% \mathrm{MgO}$ as a Function of Applied Pressure and \% Theoretical Density for $500^{\circ} \mathrm{C}$

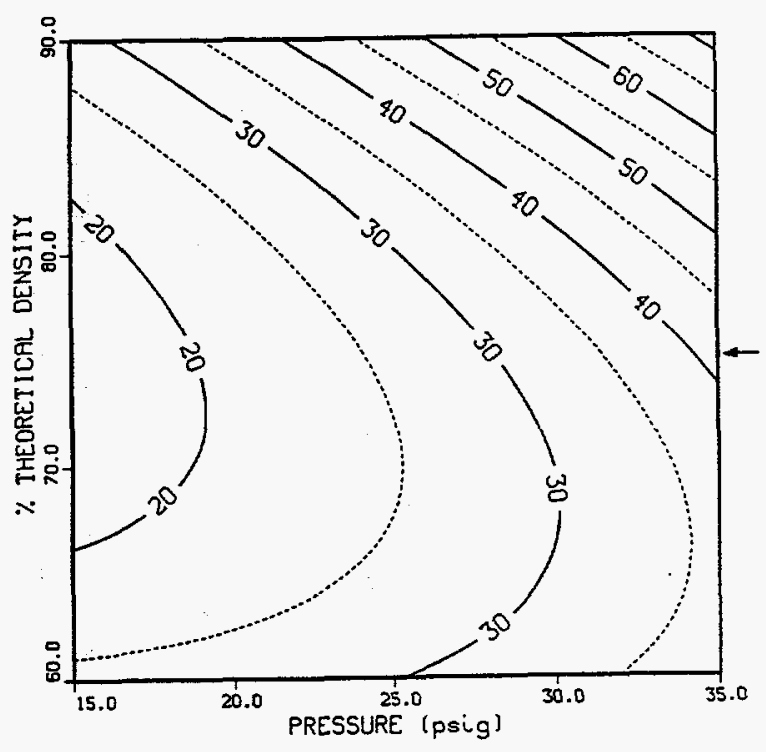

Figure 10B. Contour Plot for Estimated Deformation of EB Pellets Based on LiCl-LiBr-LiF Electrolyte and $35 \% \mathrm{MgO}$ as a Function of Applied Pressure and \% Theoretical Density for $560^{\circ} \mathrm{C}$ 
At the lower pressure, pellet deformation is in the acceptable range. At the higher pressure, however, the deformation is greater than the desired maximum of $30 \%$. The data of Figures $10 \mathrm{a}$ and $10 \mathrm{~b}$ show that the contour lines are substantially skewed to lower $\%$ TDs as the pressure is increased. This has the effect of making the separator pellet more sensitive to applied pressure for a given $\%$ TD. The optimum pellet-density design point of $\sim 74.1 \%$ TD at $15 \mathrm{psig}$ is shifted to $\sim 64.3 \% \mathrm{TD}$ at $35 \%$ psig. These data suggest that the design point should be reduced from $75 \%$ to $\sim 70 \% \mathrm{TD}$. This will reduce the maximum deformation and bring the value closer to the desired value of $30 \%$.

At $75 \%$ TD, the deformation will reach $30 \%$ at pressures of $28.0 \mathrm{psig}$ and $30 \mathrm{psig}$ at 500 ${ }^{\circ} \mathrm{C}$ and $560^{\circ} \mathrm{C}$, respectively. In comparison, at the optimum value of $69.2 \% \mathrm{TD}$, these values are predicted to $\sim 30.0 \mathrm{psig}$ and 31.9 psig-about 2 psig higher.

For critical thermal-battery application, an EB composition higher in binder than $35 \%$ $\mathrm{MgO}$ (e.g., 40\%) may be necessary to avoid excessive separator pellet deformation.

\section{Discussion}

Because of the number of possibilities that arise during the deformation process, the resulting deformation curve can be considerably different under different experimental conditions. While only the change in thickness was monitored during the deformation process, a change in the area of the pellet could also occur due to radial movement.

\section{Density Effects}

The change in deformation with increase in the level of density shows several trends. In the first case (Type A), the deformation increases with an increase in \% TD. We attribute this to an initial collapse of the primary pore structure followed by gradual radial movement of the EB material with accompanying decrease in pellet thickness. This behavior is shown by the standard EB with $35 \% \mathrm{MgO}$ and the LM\#1 EB with $25 \%$ $\mathrm{MgO}$ for all temperatures and pressures.

In the second case (Type B), the deformation exhibits a minimum with increase in $\% \mathrm{TD}$. At the lowest \% TD, the porosity is large, so that this material is expected to show a relatively large reduction in thickness once the electrolyte melts, with little radial movement of material. At intermediate densities, the relative change in thickness would be less. At the highest densities, however, the initial change in thickness would be even lower, but would be accompanied by an increase in the area due to plastic flow in the radial direction. The latter would be expected to dominate the deformation process under these conditions, resulting in the observed overall increase in deformation.

In the third case (Type $C$ ), the deformation shows a gradual decrease in deformation with increase in \% TD. At the highest \% TD, for example, the extent of radial movement is limited because of the rheology of the particular EB composition. As the porosity gradually decreases with increase in \% $\mathrm{TD}$, the degree of pore collapse becomes increasingly less, resulting in a gradual decrease in the observed change in thickness once the electrolyte melts. However, if the system experiences significant radial movement at the higher densities as a result of plastic flow, the deformation will increase again, resulting in Type $\mathrm{B}$ behavior. Type $\mathrm{C}$ behavior is shown by the all-Li EB with 30\% $\mathrm{MgO}$ and the $\mathrm{LM} \# 2 \mathrm{~EB}$ with $25 \% \mathrm{MgO}$ for all temperatures and pressures.

\section{Temperature Effects}

The reduction in deformation usually observed with increase in temperature was surprising. An increase in deformation 
would be expected based on the reduced viscosity and surface tension of the molten electrolyte at higher temperatures. For the $\mathrm{LiCl}-\mathrm{KCl}$ eutectic, for example, the viscosity is reduced from $2.23 \mathrm{mNs} / \mathrm{m}^{2}$ to $1.77 \mathrm{mNs} / \mathrm{m}^{2}$ in going from $500^{\circ} \mathrm{C}$ to $560^{\circ} \mathrm{C}$. The change in surface tension under the same conditions is $126 \mathrm{mN} / \mathrm{m}$ to 121 $\mathrm{mN} / \mathrm{m}$, respectively, not nearly as large.

The temperature effect may be related to the lower actual density of the pellet at the higher temperatures caused by expansion of the molten electrolyte. This results in a smaller change in thickness during the deformation test relative to the lower temperatures, leading to a Type $\mathrm{C}$ curve.

\section{Pressure Effects}

The effect of pressure on the deformation process was consistent for all the EBs studied. Increasing the pressure caused an increase in the observed deformation for all temperatures and densities.

\section{Rheology}

The overall observed deformation depends on the degree of pore collapse and radial movement of the EB. The relative contribution of each process determines the final type of deformation that is observed, i.e., whether the deformation response is Type $\mathrm{A}, \mathrm{B}$, or $\mathrm{C}$.

The rheology of an EB pellet under the experimental conditions of the study is very complicated and is influenced by the composition of the electrolyte as well as the MgO content. The surface tension of the electrolyte influences the wetting behavior with the $\mathrm{MgO}$ binder. This, in turn, dictates the amount of binder necessary for effective immobilization of the molten electrolyte at elevated temperatures. The conditions of EB processing (e.g., temperature, time, atmosphere, electrolyte purity, $\mathrm{MgO}$ purity, etc.) have a significant effect on the final physical properties of the resultant EB material. Changes in processing parameters are readily reflected in the deformation behavior of the pellets made from the EB mixes.

It should be emphasized that the deformation process in an actual thermal-battery environment will be different from that experienced by the pellets in the present study where the pressure and temperature were held constant. During assembly, the battery stack is subjected to a high pressure (typically 150 psig to 250 psig) to maintain good interfacial contact before being sealed into a case.

Once the battery is activated, the stack pressure relaxes immediately to a low steadystate level of only 10 psig to 40 psig. ${ }^{8}$ This relaxation process is almost entirely a result of the deformation of the separator pellet caused by the melting of the electrolyte. The temperature of the battery also changes significantly, rising on activation to some peak value and then declining until the electrolyte freezes at the end of life as a result of heat loss in the battery. Since the battery stack is tightly wrapped with glass tape and insulating ceramic blankets, the tendency for radial movement of separator material will be drastically reduced relative to an unconfined pellet.

In addition, there is always a tendency for the electrolyte to wick into the insulating wrap. This is a competition between the $\mathrm{MgO}$ and the wrap for the molten electrolyte. Because the electrolyte is already wicked into the pores of the $\mathrm{MgO}$, the tendency for de-wetting is normally minimal and only becomes a problem if the battery overheats.

Given such dynamic battery conditions, it would be very difficult to predict accurately 
the extent of pellet deformation in a thermalbattery environment. The extent of deformation will certainly be different in an actual battery compared to that observed with the deformation tester used in this work. However, the results of the present study are still useful for determining the relative impact of major changes in some critical parameters that influence the deformation process.

\section{Factor Interactions}

Varying the factors in a "one-at-a-time " approach would not show the interactions that were revealed in the approach used in this study. The strength of the statistically designed experiments lies in the ability to not only determine the relative importance of each factor, but to detect the presence of interactions as well (e.g., PD effects). The other advantage of statistically designed experiments is efficiency, i.e., the maximum amount of information is obtained in the least number of experiments.

The effect of the various factors on the deformation process is readily revealed in the coefficients for the various polynomial ex- pressions that were derived from the experimental data. These could be either positive or negative. A positive sign indicates that an increase in the independent parameter caused an increase in the deformation, while a negative sign showed a reduction in the deformation. The signs of the coefficients for the terms in the polynomial expressions for the various EBs are summarized in Table 12.

The $P, P D, D^{2}$, and $P^{2}$ coefficients were positive in all cases where that applied, as were the PD coefficients. This contrasts with the $T$ coefficients that were negative in all cases that applied. The $\mathrm{D}$ coefficient was either negative or positive, depending on the EB composition.

The relative magnitude of the coefficients indicates the importance of each term. The data in Appendices B-1 to B-8 show that the $\mathrm{D}^{2}$ term is the most important in all cases but one, with the temperature term being the least important. The pressure term was always positive, while the sign of the density term varied. Consequently, the PD interaction term was often significant at high

Table 12. Signs of Coefficients for Variables in Polynomial Expressions for Deformation of EB Pellets

\begin{tabular}{lccccccc} 
& & \multicolumn{7}{c}{ Terms } \\
\cline { 3 - 9 } Electrolyte & MgO & D & P & T & PD & P $^{2}$ & D $^{2}$ \\
\hline LiCl-KCl & 35 & + & + & NA $^{*}$ & + & + & + \\
LiCl-KCl & 40 & - & + & NA & NA & NA & NA \\
LiCl-LiBr-KBr & 25 & + & + & - & + & NA & + \\
LiCl-LiBr-KBr & 30 & - & + & - & + & NA & + \\
LiBr-KBr-LiF & 25 & + & + & - & + & NA & + \\
LiBr-KBr-LiF & 30 & - & + & - & + & NA & + \\
LiCl-LiBr-LiF & 30 & - & + & - & + & + & + \\
LiCl-LiBr-LiF & 35 & - & + & - & + & + & +
\end{tabular}

Note: $\mathrm{NA}=$ not applicable. 
positive densities, since this inhanced the pressure effect. These key points would not have been evident with the standard "one-ata-time" approach to studying the contributions of each experimental factor.

\section{Experimental Limitations}

The data generated in the present work apply for only relatively thick pellets-nominally $1.5 \mathrm{~mm}(0.059 ")$ thick. It is unknown if the same trends would hold true with separators that are used in so-called thin-cell batteries, since these separators are only 0.008 inch to 0.010 inch thick. It would be very difficult to conduct deformation tests with pellets this thin, since the absolute magnitude of the change in thickness would be very small. The tests would result in a large experimental error that would make it difficult to statistically determine the relative impact of changes in the experimental factors on the measured pellet deformation.

A deformation of $2 \%$ corresponds to a vertical displacement of 0.0011 " $(0.078 \mathrm{~mm})$. A displacement of 0.001 " (1 mil) is easily measured with good precision using $1.5-\mathrm{mm}$ thick pellets. However, for a 0.010 in-thick pellet, the same amount of deformation would result in a vertical displacement of only 0.0002 " ( 0.2 mils). Such a low value could not be measured accurately with the present experimental setup. In addition, the effect of parallelism of the pellet faces becomes increasingly important.

The present data do not address the issue of radial movement of material during the de-formation process. The present technique measures only vertical (axial) displacement of the pellet. The areal changes in the pellets would need to be measured as well to shed more light on the rheology of molten EB pellets under a wide range of experimental conditions. This would not be straight- forward, since the pellets do not always grow uniformly in the radial direction. The asymmetric growth would necessitate using some type of sophisticated areal-measurement technique (e.g., digitizing the outline of the pellet and calculating the area of the resulting shape).

\section{Optimum EB Densities and Battery- Design Implications}

The optimum conditions for pressing separator pellets to minimize excessive deformation can readily be determined by studying the contour plots and the associated regression models. The optimum densities for the various EB compositions as determined from the contour plots are summarized in Table 13 for comparison to the current design value of $75 \%$ TD. The EB compositions that are suitable for use as separators in thermal batteries are marked in Table 13 with an asterisk. These density values are the ones the thermal-battery engineer should use for prototype battery development. Only the EBs based on $\mathrm{LiCl}-\mathrm{KCl}$ eutectic and the $\mathrm{LM} \# 2$ EB with $30 \% \mathrm{MgO}$ have predicted optimum densities that are very close to the current design value of $75 \% \mathrm{TD}$.

There is a tradeoff between electrochemical performance and the mechanical properties of the EB pellets when used as separators in thermal batteries. Increasing the $\mathrm{MgO}$ content of the EBs to reduce the deformation will cause a concomitant increase in the resistivity. ${ }^{10}$

This could impact the pulse performance of the battery under a heavy load. The thermalbattery engineer will have to address this tradeoff for each particular application. This becomes most important when the battery is to be subjected to a severe environment (e.g., high levels of shock, acceleration, vibration, or spin). 
Table 13. Optimum Densities for Various EBs Examined in This Work

\begin{tabular}{lccc} 
Electrolyte & $\begin{array}{c}\text { Optimum } \\
\% \mathrm{MgO}\end{array}$ & $\begin{array}{c}\text { \% Theoretical } \\
\text { Density }\end{array}$ & $\begin{array}{c}\text { Density } \\
\text { g/cc\# }\end{array}$ \\
\hline LiCl-KCl Eutectic & $35^{*}$ & 73.4 & 1.75 \\
LiCl-KCl Eutectic & $40^{*}$ & 76.0 & 1.84 \\
LiCl-LiBr-KBr Eutectic & 25 & $\mathrm{NA}$ & $\mathrm{NA}$ \\
LiCl-LiBr-KBr Eutectic & $30^{*}$ & 69.3 & 2.11 \\
LiBr-KBr-LiF Eutectic & $25^{*}$ & 68.6 & 2.35 \\
LiBr-KBr-LiF Eutectic & $30^{*}$ & 76.1 & 2.46 \\
LiCl-LiBr-LiF Electrolyte & 30 & 70.5 & 2.19 \\
LiCl-LiBr-LiF Electrolyte & $35^{*}$ & 69.2 & 2.17 \\
& & & \\
\# Tolerance of \pm 0.05 g/cc & & \\
* Composition suitable for use as separator in thermal battery \\
\hline
\end{tabular}

\section{Conclusions}

The effects of factors such as pellet density, temperature, and applied pressure were statistically examined for their impact on the deformation of EB pellets for four electroytes with two levels of $\mathrm{MgO}$ binder. The deformation study was conducted using a statistical design-of-experiment approach that allows the effect of a relatively large number of factors to be studied with a minimal number of experiments. This approach allows the impact of each factor to be determined and to detect the presence of interactions, as well. Contour plots of the estimated response surfaces were generated over the range of experimental conditions examined in the study for purposes of interpretation.

All compositions except the $\mathrm{LiCl}-\mathrm{LiBr}-\mathrm{KBr}$ (LM\#1) eutectic with $25 \% \mathrm{MgO}$ and the LiCl-LiBr-LiF (all-Li) electrolyte with 30\% $\mathrm{MgO}$ are suitable for use as separators in thermal batteries. The study has provided estimates of the optimum densities for each of the EBs studied (Table 13). Generally (except for the all-Li mix), the variation in density dominated the deformation behavior across the various compositions. Changes in pressure also resulted in significant changes in deformation behavior; the specific effect of pressure depended on the level of pressure. Over the conditions studied, temperature seemed to have only minor effects.

The rheology of the molten EB pellets is very complex and is influenced strongly by the composition of the electrolyte and level of $\mathrm{MgO}$ binder. Three types of deformation behavior were found in the study with increase in pellet density:

- an increase in deformation (Type A curve)

- a minimum in deformation (Type B curve)

- a decrease in deformation (Type $\mathrm{C}$ curve). 
The relative behavior of the EB (type of deformation curve) is influenced by the relative contribution of pore collapse and radial movement of the EB during the deformation process.

The impact on the conductivity of the EB mix must be considered when increasing the $\mathrm{MgO}$ content of the $\mathrm{EB}$ to improve its mechanical properties when used as a separator in thermal batteries. In general, a tradeoff must be made between these two important parameters by the thermal-battery design engineer

\section{References}

1. R. A. Guidotti and F. W. Reinhardt, "Characterization of Electrolyte-Binder Mixes for Use in Thermal Batteries," SAND90-2103 (Albuquerque, NM: Sandia National Laboratories,) March 1991.

2. N. N. Volkov and L. A. Dubinskaya, "Ternary Reciprocal System of Lithium and Potassium Fluorides and Bromides," Izvestiya Fiziko-khimicheskogo Nauchnoissledovatel 'skogo instituta pri Irkutskom Gosudarstvennom universitete, 2 (1), pp. 45-47 (1953).

3. R. A. Guidotti and L. Redey, to be published.

4. D. A. Nissen, "Processing Effects on Powders for Thermal Batteries," SAND80-
8024 (Albuquerque, NM: Sandia National Laboratories), May 1980.

5. R. A. Guidotti and F. W. Reinhardt, "Characterization of DEB Powders and Pellets for $\mathrm{Ca} / \mathrm{CaCrO}{ }_{4}$ Thermal Batteries," SAND83-2270 (Albuquerque, NM: Sandia National Laboratories, July 1985).

6. R. A. Guidotti and F. W. Reinhardt, "Evaluation of Alternate Electrolytes for Use in $\mathrm{Li}(\mathrm{Si}) / \mathrm{FeS}_{2}$ Thermal Batteries," Proc. of 33rd Intern. Power Sources Symposium, pp. 369-376 (1988).

7. SAS Institute, Inc., SAS/STAT Users Guide ${ }^{\circledR}$ Cary, NC, 1989.

8. DISSPLA, Integrated Software Systems Corp., 10505 Sorento Valley Road, San Diego, CA 92121-1698 (1981).

9. R. A. Guidotti and F. W. Reinhardt, "Stack-Relaxation Studies with $\mathrm{Li}(\mathrm{Si}) / \mathrm{FeS}_{2}$ Thermal Batteries," to be published.

10. NIST Standard Reference Database 27, "NIST Properties of Molten Salts. Single Salts and Salt Mixtures Data. Density, Viscosity, Electrical Conductance, and Surface Tension," Ver. 2, National Institute of Standards and Technology, Gaithersburg, MD.

11. L. Redey, M. McParland, and R.A. Guidotti, "Resistivity Measurements of Halide/MgO Separators for Thermal Cells," Proc. of 34th Intern. Power Sources Symposium, pp. 128-131 (1990). 
Appendix A.

Deformation of EB Pellets 
.

.

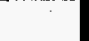


Appendix A-1. Deformation of EB Pellets Based on LiCl-KCl Eutectic

\begin{tabular}{|c|c|c|c|c|c|c|c|c|c|c|}
\hline \multirow[b]{3}{*}{ Electrolyte } & \multirow[b]{3}{*}{$\% \mathrm{MgO}$} & \multirow{3}{*}{$\begin{array}{c}\text { Density, } \\
\mathrm{g} / \mathrm{cc}\end{array}$} & \multirow{3}{*}{$\begin{array}{c}\% \\
\text { Theor. } \\
\text { Density }\end{array}$} & \multicolumn{6}{|c|}{$\%$ Change in Thickness } & \multirow{3}{*}{$\begin{array}{l}\text { Temp., } \\
\text { deg. C } \\
\end{array}$} \\
\hline & & & & \multicolumn{2}{|c|}{ (14) 14.4 psig } & \multicolumn{2}{|c|}{ (16) 23.8 psig } & \multicolumn{2}{|c|}{ (a) 32.8 psig } & \\
\hline & & & & Avg. & Std. Dev. & Avg. & Std. Dev. & Avg. & Std. Dev. & \\
\hline LiCl-KCl Eut. & 35 & 1.60 & 67.2 & -25.5 & 0.8 & -27.3 & 0.9 & -29.4 & 0.2 & 500 \\
\hline " $\quad n$ & $*$ & $1.80 *$ & 75.6 & -22.3 & 0.8 & -25.2 & 1.6 & -59.7 & 3.4 & " \\
\hline$"$ & " & 2.00 & 84.0 & -45.4 & 0.7 & -57.2 & 8.7 & -59.3 & 6.7 & • \\
\hline LiCl-KCl Eut. & 35 & 1.60 & 67.2 & -25.5 & 0.7 & -26.2 & 0.2 & -29.8 & 0.4 & 530 \\
\hline " $\quad$ " & " & $1.80 *$ & 75.6 & -21.0 & 0.9 & -23.5 & 0.3 & -60.0 & 2.4 & $"$ \\
\hline$"$ & $"$ & 2.00 & 84.0 & -42.1 & 0.7 & -51.3 & 6.2 & -61.8 & 3.7 & " \\
\hline LiCl-KCl Eut. & 35 & 1.60 & 67.2 & -24.6 & 0.8 & -26.3 & 0.4 & -33.8 & 0.8 & 560 \\
\hline " n & * & $1.80 *$ & 75.6 & -22.2 & 0.9 & -25.8 & 0.5 & -56.7 & 4.9 & " \\
\hline$"$ & $"$ & 2.00 & 84.0 & -39.6 & 1.9 & -54.5 & 6.4 & -67.0 & 7.1 & $"$ \\
\hline LiCl-KCl Eut. & 40 & 1.60 & 65.3 & -24.5 & 0.9 & -24.9 & 0.6 & -27.3 & 0.3 & 500 \\
\hline$" \quad "$ & $"$ & $1.85^{*}$ & 75.5 & -15.3 & 0.8 & -17.0 & 0.7 & -20.7 & 1.9 & $"$ \\
\hline " & " & 2.10 & 85.7 & -10.6 & 1.5 & -13.3 & 0.2 & -59.8 & 3.0 & " \\
\hline LiCl-KCl Eut. & 40 & 1.60 & 65.3 & -23.4 & 0.4 & -24.5 & 0.4 & -26.7 & 0.1 & 530 \\
\hline 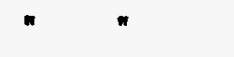 & & $1.85^{*}$ & 75.5 & -16.6 & 0.6 & -17.3 & 0.8 & -20.9 & 1.4 & $n$ \\
\hline$"$ & * & 2.10 & 85.7 & -11.3 & 1.5 & -11.3 & 1.5 & -57.6 & 4.9 & " \\
\hline LiCl-KCl Eut. & 40 & 1.60 & 65.3 & -22.8 & 0.8 & -24.6 & 1.4 & -25.7 & 1.0 & 560 \\
\hline$" \quad "$ & $"$ & $1.85 *$ & 75.5 & -16.3 & 1.4 & -16.4 & 0.3 & -17.3 & 0.3 & $"$ \\
\hline " $\quad "$ & $"$ & 2.10 & 85.7 & -12.5 & 1.0 & -13.6 & 2.0 & -54.9 & 0.5 & $n$ \\
\hline
\end{tabular}

* Design point used for thermal battery separators. 
Appendix A-2. Deformation of EB Pellets Based on LiCl-LiBr-KBr Eutectic

\begin{tabular}{|c|c|c|c|c|c|c|c|c|c|c|}
\hline \multirow[b]{3}{*}{ Electrolyte } & \multirow{3}{*}{$\% \mathrm{MgO}$} & \multirow{3}{*}{$\begin{array}{c}\text { Density, } \\
\text { g/cc }\end{array}$} & \multirow{3}{*}{$\begin{array}{c}\% \\
\text { Theor. } \\
\text { Density }\end{array}$} & \multicolumn{6}{|c|}{$\%$ Change in Thickness } & \multirow{3}{*}{$\begin{array}{l}\text { Temp., } \\
\text { deg. C }\end{array}$} \\
\hline & & & & \multicolumn{2}{|c|}{ @ 14.4 psig } & \multicolumn{2}{|c|}{ @ 23.8 psig } & \multicolumn{2}{|c|}{ Q 32.8 psig } & \\
\hline & & & & Avg. & Std. Dev. & Avg. & Std. Dev. & Avg. & Std. Dev. & \\
\hline LiCl-LiBr-KBr Eut. & 25 & 1.90 & 63.3 & -31.2 & 1.2 & -34.7 & 0.7 & -45.5 & 1.6 & 500 \\
\hline " " & - & $2.30 *$ & 76.7 & -46.2 & 1.7 & -65.1 & 1.3 & -64.2 & 5.1 & " \\
\hline " & * & 2.65 & 88.3 & -67.9 & 3.4 & -84.7 & 3.2 & -91.4 & 1.3 & " \\
\hline $\mathrm{LiCl}-\mathrm{LiBr}-\mathrm{KBr}$ Eut. & 25 & 1.90 & 63.3 & -29.6 & 1.0 & -37.2 & 1.4 & -43.3 & 1.4 & 530 \\
\hline - n & - & $2.30 *$ & 76.7 & -49.6 & 2.0 & -59.4 & 1.7 & -67.4 & 1.3 & " \\
\hline$"$ & $"$ & 2.65 & 88.3 & -64.3 & 3.1 & -75.6 & 1.4 & -90.1 & 0.6 & $n$ \\
\hline $\mathrm{LiCl}-\mathrm{LiBr}-\mathrm{KBr}$ Eut. & 25 & 1.90 & 63.3 & -29.5 & 0.9 & -34.6 & 0.5 & -43.0 & 3.1 & 560 \\
\hline 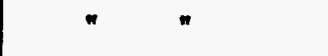 & $"$ & $2.30 *$ & 76.7 & -45.7 & 1.9 & -55.9 & 0.9 & -57.9 & 6.6 & 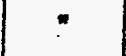 \\
\hline - & * & 2.65 & 88.3 & -55.6 & 5.4 & -74.0 & 1.4 & -86.1 & 2.1 & " \\
\hline LiCl-LiBr-KBr Eut. & 30 & 1.90 & 62.5 & -26.5 & 0.1 & -26.9 & 0.8 & -28.3 & 0.4 & 500 \\
\hline " $\quad "$ & $"$ & $2.30 *$ & 75.7 & -18.4 & 0.6 & -26.3 & 1.3 & -36.2 & 4.0 & $"$ \\
\hline$"$ & $"$ & 2.65 & 87.2 & -39.0 & 1.7 & -51.5 & 0.5 & -60.7 & 2.1 & $"$ \\
\hline LiCl-LiBr-KBr Eut. & 30 & 1.90 & 62.5 & -24.8 & 0.3 & -26.9 & 0.2 & -29.2 & 0.4 & 530 \\
\hline " & " & $2.30^{*}$ & 75.7 & -16.8 & 0.4 & -18.7 & 0.7 & -37.0 & 3.1 & $"$ \\
\hline$"$ & $"$ & 2.65 & 87.2 & -40.7 & 0.7 & -47.3 & 3.6 & -56.6 & 2.3 & " \\
\hline LiCl-LiBr-KBr Eut. & 30 & 1.90 & 62.5 & -23.3 & 0.7 & -26.8 & 0.7 & -28.3 & 1.0 & 560 \\
\hline$n \quad n$ & $\cdot$ & $2.30 *$ & 75.7 & -19.0 & 0.4 & -23.6 & 2.4 & -38.8 & 2.7 & $n$ \\
\hline$"$ & $"$ & 2.65 & 87.2 & -33.6 & 2.9 & -44.4 & 2.1 & -55.8 & 0.9 & $"$ \\
\hline
\end{tabular}

* Design point used for thermal battery separators. 
Appendix A-3. Deformation of EB Pellets Based on LiBr-KBr-LiF Eutectic

\begin{tabular}{|c|c|c|c|c|c|c|c|c|c|c|}
\hline \multirow[b]{3}{*}{ Electrolyte } & \multirow[b]{3}{*}{$\% \mathbf{M g O}$} & \multirow{3}{*}{\begin{tabular}{|c|} 
Density \\
g/cc
\end{tabular}} & \multirow{3}{*}{\begin{tabular}{|c|}
$\%$ \\
Theor. \\
Density \\
\end{tabular}} & \multicolumn{6}{|c|}{ \% Change in Thickness } & \multirow{3}{*}{$\begin{array}{l}\text { Temp., } \\
\text { deg. C }\end{array}$} \\
\hline & & & & \multicolumn{2}{|c|}{ (Q) 14.4 psig } & \multicolumn{2}{|c|}{ (1) 23.8 psig } & \multicolumn{2}{|c|}{ (1) 32.8 psig } & \\
\hline & & & & Avg. & Std. Dev. & Avg. & Std. Dev. & Avg. & Std. Dev. & \\
\hline LiBr-KBr-LiF Eut. & 25 & 1.90 & 55.6 & -24.7 & 0.3 & -27.5 & 0.7 & -31.0 & 0.7 & 500 \\
\hline " & - & $2.59 *$ & 75.7 & -14.4 & 0.3 & -18.2 & 2.4 & -25.8 & 1.0 & $\cdot$ \\
\hline$"$ & " & 2.98 & 87.1 & -31.3 & 1.9 & -45.7 & 6.4 & -60.8 & 10.6 & * \\
\hline LiBr-KBr-LiF Eut. & 25 & 1.90 & 55.6 & -24.3 & 0.6 & -27.0 & 0.4 & -30.1 & 0.3 & 530 \\
\hline " " & " & $2.59 *$ & 75.7 & -13.2 & 2.0 & -17.2 & 1.0 & -22.2 & 1.8 & $\bullet$ \\
\hline " & " & 2.98 & 87.1 & -30.3 & 1.7 & -40.9 & 3.6 & -55.6 & 6.4 & $"$ \\
\hline LiBr-KBr-LiF Eut. & 25 & 1.90 & 55.6 & -23.9 & 0.3 & -26.2 & 0.3 & -28.6 & 0.8 & 560 \\
\hline$m \cdot n$ & $\cdot$ & $2.59 *$ & 75.7 & -13.8 & 1.3 & -15.7 & 0.8 & -22.0 & 1.8 & $"$ \\
\hline$"$ & - & 2.98 & 87.1 & -27.9 & 1.6 & -41.6 & 2.4 & -45.8 & 5.0 & $"$ \\
\hline LiBr-KBr-LiF Eut. & 30 & 1.90 & 55.4 & -18.0 & 0.4 & -19.5 & 0.3 & -21.2 & 0.7 & 500 \\
\hline " " & " & $2.59 *$ & 75.5 & -11.4 & 1.4 & -10.9 & 0.2 & -12.5 & 1.1 & $"$ \\
\hline$"$ & $n$ & 2.90 & 84.5 & -8.9 & 1.6 & -10.0 & 1.1 & -16.0 & 1.9 & $"$ \\
\hline LiBr-KBr-LiF Eut. & 30 & 1.90 & 55.4 & -17.2 & 0.1 & -18.7 & 0.5 & -21.0 & 0.2 & 530 \\
\hline " & - & $2.59 *$ & 75.5 & -10.6 & 1.1 & -11.2 & 1.5 & -11.2 & 1.1 & $"$ \\
\hline$"$ & $n$ & 2.90 & 84.5 & -7.5 & 2.0 & -13.6 & 1.9 & -16.2 & 0.4 & $n$ \\
\hline LiBr-KBr-LiF Eut. & 30 & 1.90 & 55.4 & -16.0 & 0.7 & -18.0 & 0.7 & -20.9 & 0.2 & 560 \\
\hline - " & $"$ & $2.59 *$ & 75.5 & -8.8 & 1.9 & -10.7 & 1.0 & -12.6 & 1.4 & $"$ \\
\hline " & $"$ & 2.90 & 84.5 & -7.5 & 0.2 & -9.2 & 0.4 & -16.2 & 1.6 & " \\
\hline
\end{tabular}

* Design point used for thermal battery separators. 
Appendix A-4. Deformation of EB Pellets Based on LiCl-LiBr-LiF Eutectic

\begin{tabular}{|c|c|c|c|c|c|c|c|c|c|c|}
\hline \multirow[b]{3}{*}{ Electrolyte } & \multirow[b]{3}{*}{$\% \mathrm{MgO}$} & \multirow{3}{*}{$\begin{array}{c}\text { Density, } \\
\mathrm{g} / \mathrm{cc}\end{array}$} & \multirow{3}{*}{\begin{tabular}{|c|}
$\%$ \\
Theor. \\
Density \\
\end{tabular}} & \multicolumn{6}{|c|}{ \% Change in Thickness } & \multirow{3}{*}{$\begin{array}{l}\text { Temp., } \\
\text { deg. C }\end{array}$} \\
\hline & & & & \multicolumn{2}{|c|}{ Q 14.4 psig } & \multicolumn{2}{|c|}{ (1) 23.8 psig } & \multicolumn{2}{|c|}{ (a) 32.8 psig } & \\
\hline & & & & Avg. & Std. Dev. & Avg. & Std. Dev. & Avg. & Std. Dev. & \\
\hline LiCl-LiBr-LiF Eut. & 30 & 1.86 & 60.0 & -33.5 & $0.4^{\circ}$ & -41.3 & 1.4 & -52.4 & 0.8 & 500 \\
\hline " & $n$ & $2.34 *$ & 75.5 & -29.0 & 0.7 & -40.3 & 0.7 & -52.4 & 0.7 & $"$ \\
\hline " & " & 2.64 & 85.2 & -36.8 & 0.6 & -44.9 & 0.8 & -55.4 & 0.8 & " \\
\hline LiCl-LiBr-LiF Eut. & 30 & 1.86 & 60.0 & -33.8 & 0.6 & -40.2 & 0.6 & -50.1 & 1.2 & 530 \\
\hline " & $"$ & $2.34 *$ & 75.5 & -26.7 & 1.4 & -37.6 & 1.3 & -51.9 & 1.7 & $n$ \\
\hline$"$ & $n$ & 2.64 & 85.2 & -36.0 & 1.2 & -44.0 & 0.4 & -53.8 & 1.2 & $"$ \\
\hline LiCl-LiBr-LiF Eut. & 30 & 1.86 & 60.0 & -31.0 & 0.4 & -38.8 & 0.9 & -47.3 & 1.4 & 560 \\
\hline$\star$ & $\pi$ & $2.34^{*}$ & 75.5 & -24.6 & 1.8 & -37.5 & 0.8 & -47.1 & 1.8 & $"$ \\
\hline$" \quad:$ & $"$ & 2.64 & 85.2 & -36.1 & 3.9 & -44.0 & 2.4 & -52.3 & 1.5 & - \\
\hline LiCl-LiBr-LiF Eut. & 35 & 1.80 & 57.5 & -29.9 & 0.3 & -32.4 & 0.1 & -37.6 & 0.4 & 500 \\
\hline$" n$ & $n$ & $2.36^{*}$ & 75.4 & -19.5 & 3.9 & -25.0 & 0.7 & -39.8 & 2.4 & $"$ \\
\hline$"$ & $"$ & 2.66 & 85.0 & -21.7 & 1.8 & -34.6 & 3.9 & -51.1 & 3.3 & $"$ \\
\hline LiCl-LiBr-LiF Eut. & 35 & 1.80 & 57.5 & -29.5 & 0.7 & -31.7 & 0.6 & -36.6 & 0.9 & 530 \\
\hline " $\quad "$ & & $2.36^{*}$ & 75.4 & -16.8 & 1.2 & -23.4 & 0.8 & -37.6 & 1.7 & $"$ \\
\hline$"$ & $"$ & 2.66 & 85.0 & -21.3 & 2.1 & -37.5 & 4.6 & -48.8 & 4.7 & " \\
\hline LiCl-LiBr-LiF Eut. & 35 & 1.80 & 57.5 & -28.2 & 0.9 & -31.2 & 1.2 & -35.8 & 0.7 & 560 \\
\hline " $\quad "$ & $"$ & $2.36 *$ & 75.4 & -14.7 & 0.7 & -20.9 & 1.0 & -36.1 & 0.5 & $n$ \\
\hline " & $"$ & 2.66 & 85.0 & -23.7 & 5.2 & -34.1 & 4.5 & -47.5 & 4.2 & $"$ \\
\hline
\end{tabular}

* Design point used for thermal battery separators. 


\section{Appendix B. \\ Statistical Models for Deformation of EB Pellets}




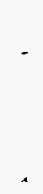

. 
The values for $P_{*}, D_{*}$, and $T_{*}$ in this appendix are obtained by scaling and centering $P, D$, and $T$, respectively, as follows:

$$
\begin{aligned}
& P_{*}=(P-23.8) / 10 \text { (psig) } \\
& D_{*}=(D-70) / 20(\% \text { TD }) \\
& T *=(T-530) / 30\left({ }^{\circ} \mathrm{C}\right)
\end{aligned}
$$


Appendix B-1. LiCl/KCl Eutectic (Std.) Electrolyte/35\% MgO*

Model

$$
\begin{array}{cc}
\log _{e}\left[\frac{Y}{100-\gamma}\right]=\beta_{0}+\beta_{1} P_{*}+\beta_{3} D *+\beta_{13} P * D * \\
\\
+\beta_{11} P^{2}+\beta_{33} D^{2}+\gamma^{2} P^{2} D^{2}+\varepsilon
\end{array}
$$

Appendix B-2. LiCl-KCI Eutectic (Std.) Electrolyte/40\% MgO

Model

$$
\begin{aligned}
& \qquad \log _{e}\left[\frac{Y}{100-Y}\right]=\beta_{0}+\beta_{1} P_{*}+\beta_{3} D *+\varepsilon \\
& \hat{\beta}_{0}=-1.309(0.012) \\
& \hat{\beta}_{1}=0.0877(0.0016) \\
& \hat{\beta}_{3}=-0.859(0.034) \\
& \quad r^{2}=0.97 \\
& \text { The values in parentheses are the standard errors of the } \\
& \text { parameter est imates. }
\end{aligned}
$$


Appendix B-3. LiCl-LiBr-KBr Eutectic (LM\#1) Electrolyte/25\% Mg0

Model

$$
\begin{aligned}
& \log _{e}\left[\frac{Y}{100-Y}\right]= \beta_{0}+\beta_{1} P *+\beta_{2} T *+\beta_{3} D * \\
&+\beta_{13} P * D *+\beta_{33} D^{2}+\varepsilon \\
& \hat{\beta}_{0}=-0.121(0.034) \hat{\beta}_{3}=1.40(0.074) \\
& \hat{\beta}_{1}=0.413(0.035) \hat{\beta}_{13}=0.374(0.082) \\
& \hat{\beta}_{2}=-0.0805(0.028) \hat{\beta} 33=0.110(0.14) \\
& r^{2}=0.98
\end{aligned}
$$

Appendix B-4. LiCl-LiBr-KBr Eutectic (LM\#1) Electrolyte/30\% MgO

Model - Same as that for Appendix B-3.

$$
\begin{array}{ll}
\hat{\beta}_{0}=-1.303(0.048) & \hat{\beta}_{3}=-0.107(0.084) \\
\hat{\beta}_{1}=0.218(0.033) & \hat{\beta}_{13}=0.243(0.055) \\
\hat{\beta}_{2}=-0.0500(0.028) & \hat{\beta}_{33}=1.82(0.16)
\end{array}
$$$$
r^{2}=0.96
$$ 
Appendix B-5. LiBr-KBr-LiF (LM\#2) Eutectic Electrolyte/25\% Mg0

\section{Model}

$$
\begin{array}{ll}
\log _{e}\left[\frac{Y}{100-Y}\right]= & \beta_{0}+\beta_{1} P_{*}+\beta_{2} T_{*}+\beta_{3} D * \\
& +\beta_{13} P_{*} D *+\beta_{33} D^{2}+\varepsilon \\
\hat{\beta}_{0}=-1.710(0.024) & \hat{\beta}_{3}=0.186(0.018) \\
\hat{\beta}_{1}=0.321(0.016) & \hat{\beta}_{13}=0.233(0.024) \\
\hat{\beta}_{2}=-0.0588(0.012) & \hat{\beta}_{33}=1.637(0.055) \\
& r^{2}=0.98
\end{array}
$$

Appendix B-6. LiBr-KBr-LiF Eutectic (LM\#2) Electrolyte/30\% MgO

Model - Same as that for Appendix B-5.

$$
\begin{array}{ll}
\hat{\beta}_{0}=-2.035(0.054) & \hat{\beta}_{3}=-0.395(0.038) \\
\hat{\beta}_{1}=0.222(0.030) & \hat{\beta}_{13}=0.132(0.045) \\
\hat{\beta}_{2}=-0.0378(0.024) & \hat{\beta}_{33}=0.550(0.123) \\
r^{2}=0.92
\end{array}
$$


Appendix B-7. LiCl-LiBr-LiF Eutectic (All-Li) Electrolyte/30\% Mg0

\section{Model}

$$
\begin{array}{cl}
\log _{e}\left[\frac{\gamma}{100-\gamma}\right]= & \beta_{0}+\beta_{1} P_{*}+\beta_{2} T *+\beta_{3} D * \\
& +\beta_{13} P_{*} D * \beta_{11} P^{2}+\beta_{33} D^{2}+\varepsilon \\
\hat{\beta}_{0}=-0.509(0.027) & \hat{\beta}_{13}=0.0299(0.031) \\
\hat{\beta}_{1}=0.434(0.017) & \hat{\beta}_{11}=0.0372(0.030) \\
\hat{\beta}_{2}=-0.0673(0.016) & \hat{\beta}_{33}=0.465(0.072) \\
\hat{\beta}_{3}=-0.0130(0.027) & \\
& r^{2}=0.98
\end{array}
$$

Appendix B-8. LiCl-LiBr-Lif Eutectic (All-Li) Electrolyte/35\% Mg0

Model - Same as that for Appendix B-7.

$$
\begin{array}{lll}
\hat{\beta}_{0}=-1.224(0.026) & \hat{\beta}_{13}=0.445(0.029) \\
\hat{\beta}_{1}=0.471(0.017) & \hat{\beta}_{11}=0.0981(0.026) \\
\hat{\beta}_{2}=-0.0514(0.013) & \hat{\beta}_{33}=1.020(0.081) \\
\hat{\beta}_{3}=-0.0603(0.025) & \\
& r^{2}=0.98
\end{array}
$$



Appendix C.

Comparison of Predicted and Experimental Values

for the Deformation of EB Pellets 


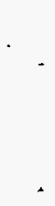


Appendix C-1. LiCl-KCl Eutectic (Std.) Electrolyte/35\% Mg0

Obs. \% Est. \%

Std.

Change Change Diff. Dev.

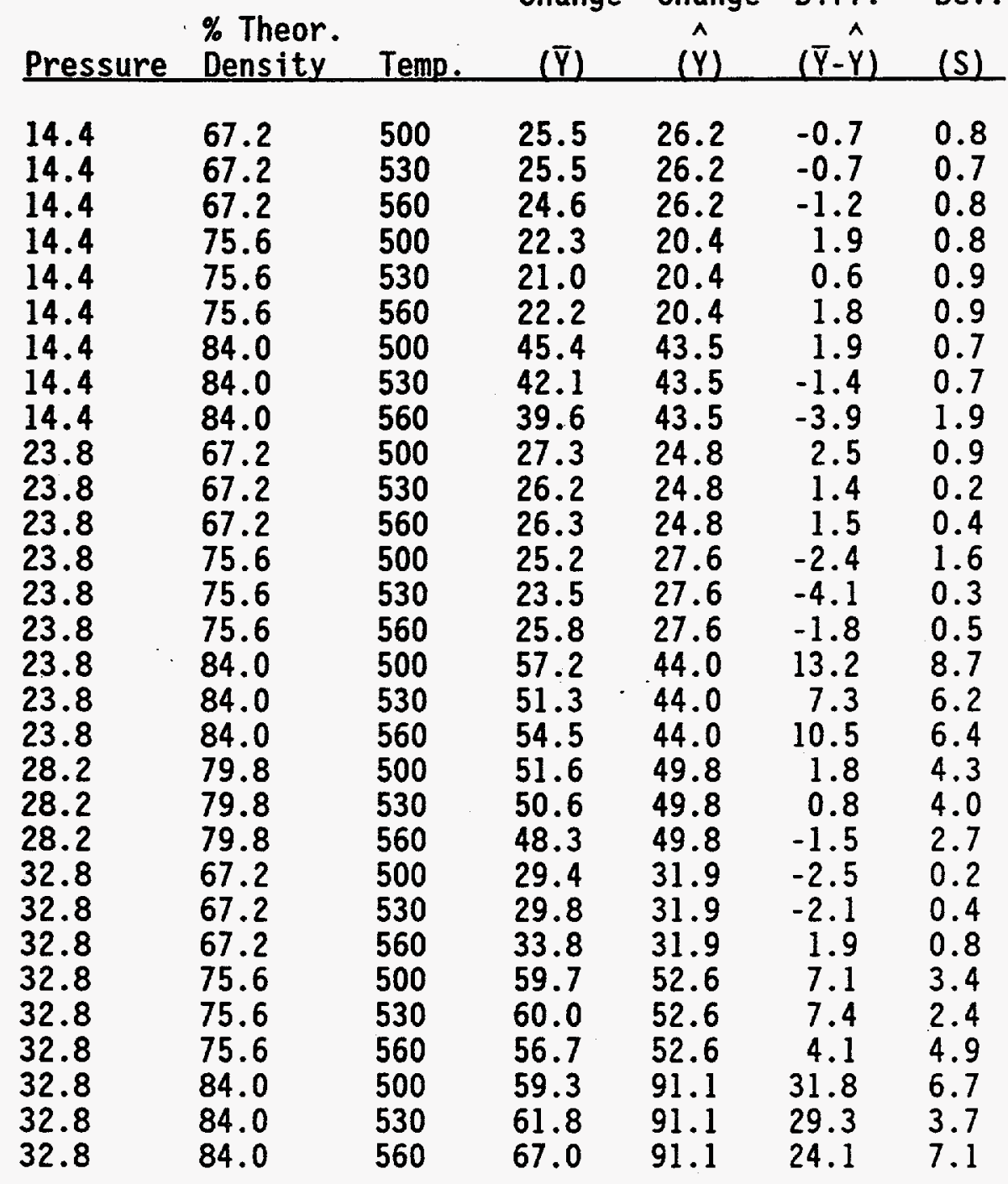


Appendix C-2. LiCl-KCl Eutectic Electrolyte/40\% MgO

\begin{tabular}{|c|c|c|c|c|c|c|}
\hline Pressure & $\begin{array}{l}\% \text { Theor. } \\
\text { Density }\end{array}$ & Temp. & $\begin{array}{c}\text { Obs. \% } \\
\text { Change } \\
(\bar{Y})\end{array}$ & $\begin{array}{c}\text { Est. \% } \\
\text { Change } \\
\hat{(Y)}\end{array}$ & $\begin{array}{l}\text { Diff. } \\
(\bar{Y}-\hat{Y})\end{array}$ & $\begin{array}{l}\text { Std. } \\
\text { Dev. }\end{array}$ \\
\hline $\begin{array}{l}14.4 \\
14.4 \\
14.4 \\
14.4 \\
14.4 \\
14.4 \\
14.4 \\
14.4 \\
14.4 \\
23.8 \\
23.8 \\
23.8 \\
23.8 \\
23.8 \\
23.8 \\
23.8 \\
23.8 \\
23.8 \\
28.2 \\
28.2 \\
28.2 \\
32.8 \\
32.8 \\
32.8 \\
32.8 \\
32.8 \\
32.8 \\
32.8 \\
32.8 \\
32.8\end{array}$ & $\begin{array}{l}65.3 \\
65.3 \\
65.3 \\
75.5 \\
75.5 \\
75.5 \\
85.7 \\
85.7 \\
85.7 \\
65.3 \\
65.3 \\
65.3 \\
75.5 \\
75.5 \\
75.5 \\
85.7 \\
85.7 \\
85.7 \\
80.0 \\
80.0 \\
80.0 \\
65.3 \\
65.3 \\
65.3 \\
75.5 \\
75.5 \\
75.5 \\
85.7 \\
85.7 \\
85.7\end{array}$ & $\begin{array}{l}500 \\
530 \\
560 \\
500 \\
530 \\
560 \\
500 \\
530 \\
560 \\
500 \\
530 \\
560 \\
500 \\
530 \\
560 \\
500 \\
530 \\
560 \\
500 \\
530 \\
560 \\
500 \\
530 \\
560 \\
500 \\
530 \\
560 \\
500 \\
530 \\
560\end{array}$ & $\begin{array}{l}24.5 \\
23.4 \\
22.8 \\
15.3 \\
16.6 \\
16.3 \\
10.6 \\
11.3 \\
12.5 \\
24.9 \\
24.5 \\
24.6 \\
17.0 \\
17.3 \\
16.4 \\
13.3 \\
11.3 \\
13.6 \\
14.8 \\
14.7 \\
15.2 \\
27.3 \\
26.7 \\
25.7 \\
20.7 \\
20.9 \\
17.3 \\
59.8 \\
57.6 \\
54.9\end{array}$ & $\begin{array}{l}23.3 \\
23.3 \\
23.3 \\
16.4 \\
16.4 \\
16.4 \\
11.2 \\
11.2 \\
11.2 \\
24.8 \\
24.8 \\
24.8 \\
17.6 \\
17.6 \\
17.6 \\
12.1 \\
12.1 \\
12.1 \\
15.4 \\
15.4 \\
15.4 \\
26.3 \\
26.3 \\
26.3 \\
18.7 \\
18.7 \\
18.7 \\
13.0 \\
13.0 \\
13.0\end{array}$ & $\begin{array}{r}1.2 \\
0.1 \\
-0.5 \\
-1.1 \\
0.2 \\
-0.1 \\
-0.6 \\
0.1 \\
1.3 \\
0.1 \\
-0.3 \\
-0.2 \\
-0.6 \\
-0.3 \\
-1.2 \\
1.2 \\
-0.8 \\
1.5 \\
-0.6 \\
-0.7 \\
-0.2 \\
1.0 \\
0.4 \\
-0.6 \\
2.0 \\
2.2 \\
-1.4 \\
46.8 \\
44.6 \\
41.9\end{array}$ & $\begin{array}{l}0.9 \\
0.4 \\
0.8 \\
0.8 \\
0.6 \\
1.4 \\
1.5 \\
1.5 \\
1.0 \\
0.6 \\
0.4 \\
1.4 \\
0.7 \\
0.8 \\
0.3 \\
0.2 \\
1.5 \\
2.0 \\
1.4 \\
0.6 \\
0.7 \\
0.3 \\
0.1 \\
1.0 \\
1.9 \\
1.4 \\
0.3 \\
3.0 \\
4.9 \\
0.5\end{array}$ \\
\hline
\end{tabular}


Appendix C-3. LiCl-LiBr-KBr Eutectic (LM\#1) Electrolyte/25\% MgO

\begin{tabular}{|c|c|c|c|c|c|c|}
\hline Pressure & $\begin{array}{l}\text { \% Theor. } \\
\text { Density }\end{array}$ & Temp. & $\begin{array}{c}\text { Obs. \% } \\
\text { Change } \\
(\bar{Y})\end{array}$ & $\begin{array}{c}\text { Est. \% } \\
\text { Change } \\
\hat{(Y)} \\
\end{array}$ & $\begin{array}{l}\text { Diff. } \\
(\bar{Y}-\hat{Y})\end{array}$ & $\begin{array}{l}\text { Std } \\
\text { Dev } \\
(S)\end{array}$ \\
\hline $\begin{array}{l}14.4 \\
14.4 \\
14.4 \\
14.4 \\
14.4 \\
14.4 \\
14.4 \\
14.4 \\
14.4 \\
23.8 \\
23.8 \\
23.8 \\
23.8 \\
23.8 \\
23.8 \\
23.8 \\
23.8 \\
23.8 \\
32.8 \\
32.8 \\
32.8 \\
32.8 \\
32.8 \\
32.8 \\
32.8 \\
32.8\end{array}$ & $\begin{array}{l}63.3 \\
63.3 \\
63.3 \\
76.7 \\
76.7 \\
76.7 \\
88.3 \\
88.3 \\
88.3 \\
63.3 \\
63.3 \\
63.3 \\
76.7 \\
76.7 \\
76.7 \\
88.3 \\
88.3 \\
88.3 \\
63.3 \\
63.3 \\
63.3 \\
76.7 \\
76.7 \\
76.7 \\
88.3 \\
88.3 \\
88.3\end{array}$ & $\begin{array}{l}500 \\
530 \\
560 \\
500 \\
530 \\
560 \\
500 \\
530 \\
560 \\
500 \\
530 \\
560 \\
500 \\
530 \\
560 \\
500 \\
530 \\
560 \\
500 \\
530 \\
560 \\
500 \\
530 \\
560 \\
500 \\
530 \\
560\end{array}$ & $\begin{array}{l}31.2 \\
29.6 \\
29.5 \\
46.2 \\
49.6 \\
45.7 \\
67.9 \\
64.3 \\
55.6 \\
34.7 \\
37.2 \\
34.6 \\
65.1 \\
59.4 \\
55.9 \\
84.7 \\
75.6 \\
74.0 \\
45.5 \\
43.3 \\
43.0 \\
64.2 \\
67.4 \\
57.9 \\
91.4 \\
90.1 \\
86.1\end{array}$ & $\begin{array}{l}31.7 \\
30.0 \\
28.3 \\
48.3 \\
46.3 \\
44.3 \\
65.0 \\
63.2 \\
61.3 \\
37.8 \\
36.0 \\
34.1 \\
60.8 \\
58.9 \\
56.9 \\
79.1 \\
77.7 \\
76.3 \\
44.1 \\
42.1 \\
40.2 \\
71.6 \\
69.9 \\
68.2 \\
88.2 \\
87.3 \\
86.4\end{array}$ & $\begin{array}{r}-0.5 \\
-0.4 \\
1.2 \\
-2.1 \\
3.3 \\
1.4 \\
2.9 \\
1.1 \\
-5.7 \\
-3.1 \\
1.2 \\
0.5 \\
4.3 \\
0.5 \\
-1.0 \\
5.6 \\
-2.1 \\
-2.3 \\
1.4 \\
1.2 \\
2.8 \\
-7.4 \\
-2.5 \\
-10.3 \\
3.2 \\
2.8 \\
-0.3\end{array}$ & $\begin{array}{l}1.2 \\
1.0 \\
0.9 \\
1.7 \\
2.0 \\
1.9 \\
3.4 \\
3.1 \\
5.4 \\
0.7 \\
1.4 \\
0.5 \\
1.3 \\
1.7 \\
0.9 \\
3.2 \\
1.4 \\
1.4 \\
1.6 \\
1.4 \\
3.1 \\
5.1 \\
1.3 \\
6.6 \\
1.3 \\
0.6 \\
2.1\end{array}$ \\
\hline
\end{tabular}


Appendix C-4. LiCl-LiBr-KBr Eutectic (LM\#1) Electrolyte/30\% Mg0

Obs. \% Est. \% Std. Change Change Diff. Dev.

Pressure Density Temp. $(\bar{Y}) \quad(\hat{Y}) \quad(\bar{Y}-\hat{Y}) \quad(S)$

$\begin{array}{rrrrrrr}14.4 & 62.5 & 500 & 26.5 & 25.4 & 1.1 & 0.1 \\ 14.4 & 62.5 & 530 & 24.8 & 24.5 & 0.3 & 0.3 \\ 14.4 & 62.5 & 560 & 23.3 & 23.6 & -0.3 & 0.7 \\ 14.4 & 75.7 & 500 & 18.4 & 19.7 & -1.3 & 0.6 \\ 14.4 & 75.7 & 530 & 16.8 & 18.9 & -2.1 & 0.4 \\ 14.4 & 75.7 & 560 & 19.0 & 18.2 & 0.8 & 0.4 \\ 14.4 & 87.2 & 500 & 39.0 & 40.2 & -1.2 & 1.7 \\ 14.4 & 87.2 & 530 & 40.7 & 39.0 & 1.7 & 0.7 \\ 14.4 & 87.2 & 560 & 33.6 & 37.8 & -4.2 & 2.9 \\ 23.8 & 62.5 & 500 & 26.9 & 27.8 & -0.9 & 0.8 \\ 23.8 & 62.5 & 530 & 26.9 & 26.8 & 0.1 & 0.2 \\ 23.8 & 62.5 & 560 & 26.8 & 25.8 & 1.0 & 0.7 \\ 23.8 & 75.7 & 500 & 26.3 & 24.3 & 2.0 & 1.3 \\ 23.8 & 75.7 & 530 & 18.7 & 23.4 & -4.7 & 0.7 \\ 23.8 & 75.7 & 560 & 23.6 & 22.5 & 1.1 & 2.4 \\ 23.8 & 87.2 & 500 & 51.5 & 50.1 & 1.4 & 0.5 \\ 23.8 & 87.2 & 530 & 47.3 & 48.8 & -1.5 & 3.6 \\ 23.8 & 87.2 & 560 & 44.4 & 47.6 & -3.2 & 2.1 \\ 32.8 & 62.5 & 500 & 28.3 & 30.1 & -1.8 & 0.4 \\ 32.8 & 62.5 & 530 & 29.2 & 29.1 & 0.1 & 0.4 \\ 32.8 & 62.5 & 560 & 28.3 & 28.0 & 0.3 & 1.0 \\ 32.8 & 75.7 & 500 & 36.2 & 29.4 & 6.8 & 4.0 \\ 32.8 & 75.7 & 530 & 37.0 & 28.4 & 8.6 & 3.1 \\ 32.8 & 75.7 & 560 & 38.8 & 27.4 & 11.4 & 2.7 \\ 32.8 & 87.2 & 500 & 60.7 & 59.6 & 1.1 & 2.1 \\ 32.8 & 87.2 & 530 & 56.6 & 58.4 & -1.8 & 2.3 \\ 32.8 & 87.2 & 560 & 55.8 & 57.2 & -1.4 & 0.9\end{array}$


Appendix C-5. LiBr-KBr-LiF Eutectic (LM\#2) Electrolyte/25\% Mg0

\begin{tabular}{lllllrr} 
& \multicolumn{7}{c}{$\begin{array}{c}\text { Obs. \% } \\
\text { Change }\end{array}$} & $\begin{array}{c}\text { Est. } \% \\
\text { Change } \\
\text { Diff. }\end{array}$ & $\begin{array}{r}\text { Std. } \\
\text { Dev. }\end{array}$ \\
Pressure & $\begin{array}{l}\text { \% Theor. } \\
\text { Density }\end{array}$ & Temp. & $(\bar{Y})$ & $(Y)$ & $(\bar{Y}-\hat{Y})$ & $(S)$ \\
\hline 14.4 & 55.6 & 500 & 24.7 & 25.3 & -0.6 & 0.3 \\
14.4 & 55.6 & 530 & 24.3 & 24.2 & 0.1 & 0.6 \\
14.4 & 55.6 & 560 & 23.9 & 23.2 & 0.7 & 0.3 \\
14.4 & 75.7 & 500 & 14.4 & 13.8 & 0.6 & 0.3 \\
14.4 & 75.7 & 530 & 13.2 & 13.1 & 0.1 & 2.0 \\
14.4 & 75.7 & 560 & 13.8 & 12.5 & 1.3 & 1.3 \\
14.4 & 87.1 & 500 & 31.3 & 31.3 & 0.0 & 1.9 \\
14.4 & 87.1 & 530 & 30.3 & 30.1 & 0.2 & 1.7 \\
14.4 & 87.1 & 560 & 27.9 & 28.9 & -1.0 & 1.6 \\
23.8 & 55.6 & 500 & 27.5 & 28.2 & -0.7 & 0.7 \\
23.8 & 55.6 & 530 & 27.0 & 27.0 & 0.0 & 0.4 \\
23.8 & 55.6 & 560 & 26.2 & 25.9 & 0.3 & 0.3 \\
23.8 & 75.7 & 500 & 18.2 & 18.8 & -0.6 & 2.4 \\
23.8 & 75.7 & 530 & 17.2 & 17.9 & -0.7 & 1.0 \\
23.8 & 75.7 & 560 & 15.7 & 17.0 & -1.3 & 0.8 \\
23.8 & 87.1 & 500 & 45.7 & 42.7 & 3.0 & 6.4 \\
23.8 & 87.1 & 530 & 40.9 & 41.2 & -0.3 & 3.6 \\
23.8 & 87.1 & 560 & 41.6 & 39.8 & 1.8 & 2.4 \\
32.8 & 55.6 & 500 & 31.0 & 31.1 & -0.1 & 0.7 \\
32.8 & 55.6 & 530 & 30.1 & 29.8 & 0.3 & 0.3 \\
32.8 & 55.6 & 560 & 28.6 & 28.6 & 0.0 & 0.8 \\
32.8 & 75.7 & 500 & 25.8 & 24.7 & 1.1 & 1.0 \\
32.8 & 75.7 & 530 & 22.2 & 23.6 & -1.4 & 1.8 \\
32.8 & 75.7 & 560 & 22.0 & 22.6 & -0.6 & 1.8 \\
32.8 & 87.1 & 500 & 60.8 & 54.3 & 6.5 & 10.6 \\
32.8 & 87.1 & 530 & 55.6 & 52.9 & 2.7 & 6.4 \\
32.8 & 87.1 & 560 & 45.8 & 51.4 & -5.6 & 5.0
\end{tabular}


Appendix C-6. LiBr-KBr-LiF Eutectic (LM\#2) Electrolyte/30\% Mg0

\begin{tabular}{|c|c|c|c|c|c|c|}
\hline Pressure & $\begin{array}{l}\text { \% Theor. } \\
\text { Density }\end{array}$ & Temp. & $\begin{array}{l}\text { Obs. \% } \\
\text { Change } \\
(\bar{Y})\end{array}$ & $\begin{array}{l}\text { Est. \% } \\
\text { Change } \\
\hat{(Y)}\end{array}$ & $\begin{array}{l}\text { Diff. } \\
(\bar{Y}-\hat{Y})\end{array}$ & $\begin{array}{l}\text { Std. } \\
\text { Dev. }\end{array}$ \\
\hline $\begin{array}{l}14.4 \\
14.4 \\
14.4 \\
14.4 \\
14.4 \\
14.4 \\
14.4 \\
14.4 \\
14.4 \\
23.8 \\
23.8 \\
23.8 \\
23.8 \\
23.8 \\
23.8 \\
23.8 \\
23.8 \\
23.8 \\
32.8 \\
32.8 \\
32.8 \\
32.8 \\
32.8 \\
32.8 \\
32.8 \\
32.8\end{array}$ & $\begin{array}{l}55.4 \\
55.4 \\
55.4 \\
75.5 \\
75.5 \\
75.5 \\
84.5 \\
84.5 \\
84.5 \\
55.4 \\
55.4 \\
55.4 \\
75.5 \\
75.5 \\
75.5 \\
84.5 \\
84.5 \\
84.5 \\
55.4 \\
55.4 \\
55.4 \\
75.5 \\
75.5 \\
75.5 \\
84.5 \\
84.5\end{array}$ & $\begin{array}{l}500 \\
530 \\
560 \\
500 \\
530 \\
560 \\
500 \\
530 \\
560 \\
500 \\
530 \\
560 \\
500 \\
530 \\
560 \\
500 \\
530 \\
560 \\
500 \\
530 \\
560 \\
500 \\
530 \\
560 \\
500 \\
530 \\
560\end{array}$ & $\begin{array}{r}18.0 \\
17.2 \\
16.0 \\
11.4 \\
10.6 \\
8.8 \\
8.9 \\
7.5 \\
7.5 \\
19.5 \\
18.7 \\
18.0 \\
10.9 \\
11.2 \\
10.7 \\
10.0 \\
13.6 \\
9.2 \\
21.2 \\
21.0 \\
20.9 \\
12.5 \\
11.2 \\
12.6 \\
16.0 \\
16.2 \\
16.2\end{array}$ & $\begin{array}{r}17.7 \\
17.2 \\
16.7 \\
9.1 \\
8.7 \\
8.4 \\
9.2 \\
8.9 \\
8.6 \\
19.5 \\
18.9 \\
18.4 \\
11.3 \\
10.9 \\
10.5 \\
12.0 \\
11.6 \\
11.2 \\
21.4 \\
20.7 \\
20.1 \\
13.8 \\
13.4 \\
12.9 \\
15.3 \\
14.8 \\
14.4\end{array}$ & $\begin{array}{r}0.3 \\
0.0 \\
-0.7 \\
2.3 \\
1.9 \\
0.4 \\
-0.3 \\
-1.4 \\
-1.1 \\
0.0 \\
-0.2 \\
-0.4 \\
-0.4 \\
0.3 \\
0.2 \\
-2.0 \\
2.0 \\
-2.0 \\
-0.2 \\
0.3 \\
0.8 \\
-1.3 \\
-2.2 \\
-0.3 \\
0.7 \\
1.4 \\
1.8\end{array}$ & $\begin{array}{l}0.4 \\
0.7 \\
0.2 \\
0.2 \\
1.1 \\
1.1 \\
1.4 \\
1.9 \\
0.4 \\
1.6\end{array}$ \\
\hline
\end{tabular}


Appendix C-7. LiCl-LiBr-LiF Eutectic (All-Li) Electrolyte/30\% Mg0

\begin{tabular}{|c|c|c|c|c|c|c|}
\hline Pressure & $\begin{array}{l}\% \text { Theor. } \\
\text { Density }\end{array}$ & Temp. & $\begin{array}{c}\text { Obs. \% } \\
\text { Change } \\
(\bar{Y})\end{array}$ & $\begin{array}{c}\text { Est. \% } \\
\text { Change } \\
\hat{(Y)}\end{array}$ & $\begin{array}{l}\text { Diff. } \\
(\bar{Y}-\hat{Y})\end{array}$ & $\begin{array}{l}\text { Std. } \\
\text { Dev. } \\
(S)\end{array}$ \\
\hline $\begin{array}{l}14.4 \\
14.4 \\
14.4 \\
14.4 \\
14.4 \\
14.4 \\
14.4 \\
14.4 \\
14.4 \\
23.8 \\
23.8 \\
23.8 \\
23.8 \\
23.8 \\
23.8 \\
23.8 \\
23.8 \\
23.8 \\
32.8 \\
32.8 \\
32.8 \\
32.8 \\
32.8 \\
32.8 \\
32.8 \\
32.8 \\
32.8\end{array}$ & $\begin{array}{l}60.0 \\
60.0 \\
60.0 \\
75.5 \\
75.5 \\
75.5 \\
85.2 \\
85.2 \\
85.2 \\
60.0 \\
60.0 \\
60.0 \\
75.5 \\
75.5 \\
75.5 \\
85.2 \\
85.2 \\
85.2 \\
60.0 \\
60.0 \\
60.0 \\
75.5 \\
75.5 \\
75.5 \\
85.2 \\
85.2 \\
85.2\end{array}$ & $\begin{array}{l}500 \\
530 \\
560 \\
500 \\
530 \\
560 \\
500 \\
530 \\
560 \\
500 \\
530 \\
560 \\
500 \\
530 \\
560 \\
500 \\
530 \\
560 \\
500 \\
530 \\
560 \\
500 \\
530 \\
560 \\
500 \\
530 \\
560\end{array}$ & $\begin{array}{l}33.5 \\
33.8 \\
31.0 \\
29.0 \\
26.7 \\
24.6 \\
36.8 \\
36.0 \\
36.1 \\
41.3 \\
40.2 \\
38.8 \\
40.3 \\
37.6 \\
37.5 \\
44.9 \\
44.0 \\
44.0 \\
52.4 \\
50.1 \\
47.3 \\
52.4 \\
51.9 \\
47.1 \\
55.4 \\
53.8 \\
52.3\end{array}$ & $\begin{array}{l}33.6 \\
32.1 \\
30.7 \\
31.2 \\
29.7 \\
28.3 \\
35.9 \\
34.4 \\
32.9 \\
42.1 \\
40.5 \\
38.9 \\
39.9 \\
38.3 \\
36.7 \\
45.5 \\
43.8 \\
42.1 \\
52.2 \\
50.5 \\
48.9 \\
50.5 \\
48.8 \\
47.1 \\
56.4 \\
54.8 \\
53.1\end{array}$ & $\begin{array}{r}-0.1 \\
1.7 \\
0.3 \\
-2.2 \\
-3.0 \\
-3.7 \\
0.9 \\
1.6 \\
3.2 \\
-0.8 \\
-0.3 \\
-0.1 \\
0.4 \\
-0.7 \\
0.8 \\
-0.6 \\
0.2 \\
1.9 \\
0.2 \\
-0.4 \\
-1.6 \\
1.9 \\
3.1 \\
0.0 \\
-1.0 \\
-1.0 \\
-0.8\end{array}$ & $\begin{array}{l}0.4 \\
0.6 \\
0.4 \\
0.7 \\
1.4 \\
1.8 \\
0.6 \\
1.2 \\
3.9 \\
1.4 \\
0.6 \\
0.9 \\
0.7 \\
1.3 \\
0.8 \\
0.8 \\
0.4 \\
2.4 \\
0.8 \\
1.2 \\
1.4 \\
0.7 \\
1.7 \\
1.8 \\
0.8 \\
1.2 \\
1.5\end{array}$ \\
\hline
\end{tabular}


Appendix C-8. LiCl-LiBr-Lif Eutectic (A11-Li) Electrolyte/35\% Mg0

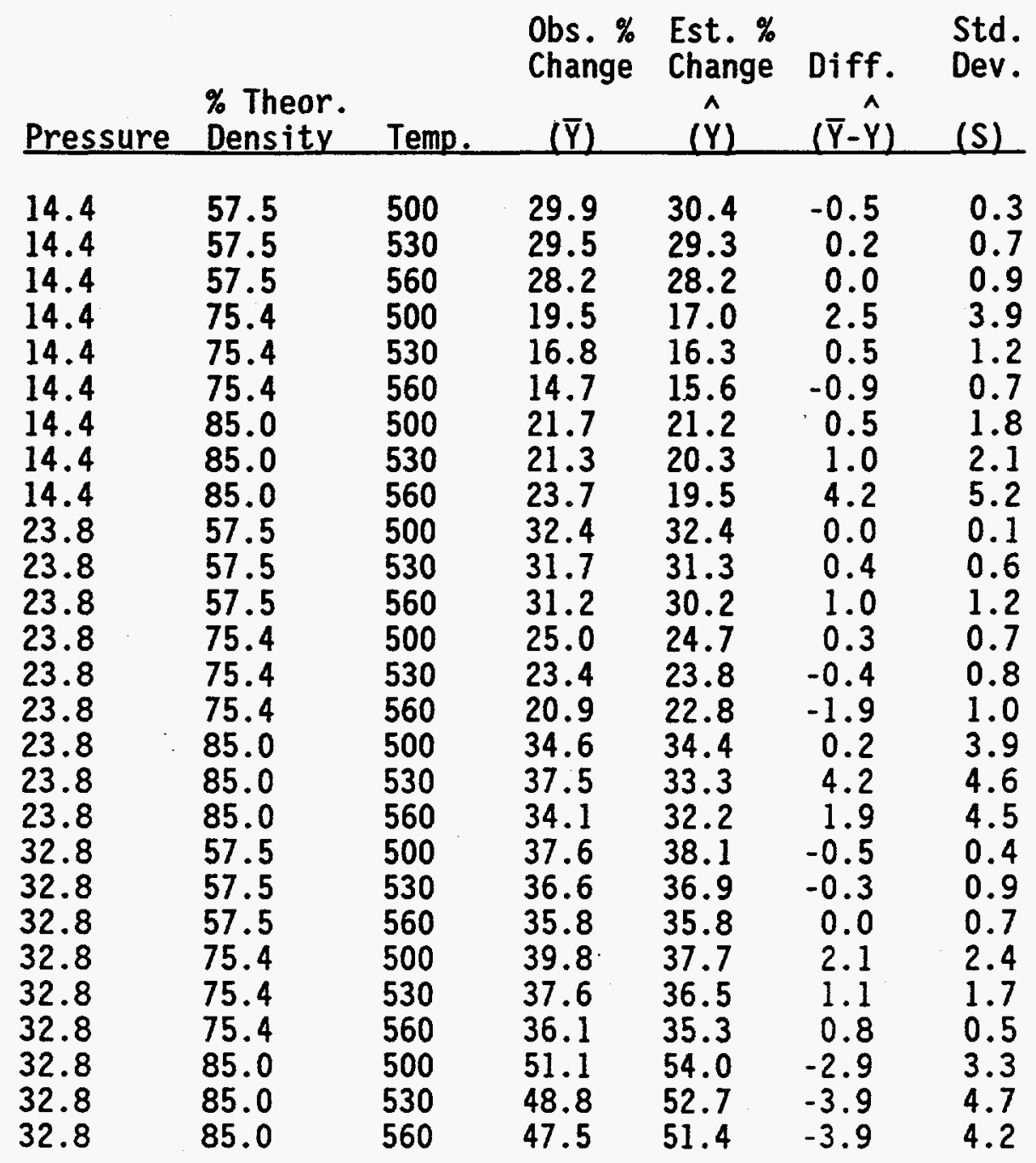



Unlimited Distribution

2 Wright Laboratory

Aero Propulsion and Power Directorate

Att: R A. Marsh

D. M. Ryan

WL/POOS-2

1950 Fifth St.

Wright-Paterson AFB, OH 45433-7251

4 Argonne National Laboratory

Chemical Technology Div.

Attn: D. Vissers

L. Redey

J. Smaga

T. Kaun

9700 South Cass Ave.

Argonne, IL 604339

2 Army Materials and Mechanism

Research Center

Attn: J. W. McCauley

P. Wong

Watertown, MA 02172

2 Army Research Lab

Attn: F. Krieger

A. Goldberg

2800 Powder Mill Road

Adelphi, MD. 20783

1 A. A. Benderly, Consultant 9915 Logan Dr.

Potomac, Maryland 20854

3 Boeing Aerospace

Attn:

C. Johnson

J. Kavandi

Sid Gross

P. O. Box 3999

Seattle, WA 98124
5 Eagle-Picher Industries, Inc.

Electronics Div. Couples Dept.

Attn: R. Spencer

F. Smith

J. DeGruson

R. Hudson

J. Wells

P.O. Box 47

Joplin, MO 64802

2 General Dynamics Pomona Div.

Attn: Kathy Wong Jaime Perez

Mail Zone 4-66

P. O. Box 2507

Pomona, CA 91769-2507

1 Hughes Aircraft Co.

Attn: L. A. Schaum, Jr.

Building 803

Mail Station E

P. O. Box 11337

Tuscon Az. 85721

1 Martin-Marietta

Specialty Components Div.

Attn: W. McCracken

P.O. Box 2908

Largo, FL 34649

1 Naval Ordnance Station

Attn: K. Englander

Code 5123C

Indian Head, MD 20640

2 Naval Sea Systems Command

Attn: F. Butler

M. F. Murphy

Attention Code SEA63R-32

Washington, DC 20235 
2 Naval Surface Warfare Center

Attn: B. Larrick

C. E. Mueller

Silver Springs, MD. 20910

1 Naval Surface Warfare Center

Attn: C. Winchester

Code R33

10901 New Hampshire Ave.

Silver Spring, MD 20903-5000

1 Naval Underwater Systems Center

Attn: R. S. Lazar

Code 36301

Newport, RI 02840

3 Naval Weapons Center

Attn: M. H. Miles

R. Nolan (Code 3626)

D. Rosenlof (Code 3626)

China Lake, Ca 93555

1 Parker Hannifin

Attn: Arvind Ahluwalia, MS K141

14300 Alton Parkway

Irvine, CA 92718-1814

2 SAFT America

Attn: K. K. Press

J. Embrey

107 Beaver Court

Cockeysville, MD 21030
1 Technochem Co.

Attn: Shyam Argade

203A Creekridge Rd.

Greensboro, NC 27406

2 Defence Research Agency

Materials Dept.

Attn: J. Knight

A. G. Ritchie

Building R178

Famborough

Hants GU14 6TD

ENGLAND

1 Leclanche, S.A.

Attn: P. Reutschi

48, Avenue de Grandson

CH-1401 Yverdon-Les-Bain

SWITZERLAND

10614 A. H. Andazola, 2222

10614 J. A. Gilbert, 2222

10614 K. R. Grothaus, 2222

100614 R. A Guidotti, 2223

10614 F. P. Lasky, 2222

10614 L. M. Moya, 2222

10614 F. W. Reinhardt,2223

10614 G. L. Scharrer, 2223

$50829 \quad$ E. V. Thomas, 12323

19018 Central Technical Files, 8523-2

50899 Technical Library, 13414

10619 Print Media, 12613

20100 Document Processing, 7613-2

For DOE/OSTI 